\title{
Learning Graph Representation with Randomized Neural Network for Dynamic Texture Classification
}

\author{
Lucas C. Ribas ${ }^{a, b, c}$, Jarbas Joaci de Mesquita Sá Junior ${ }^{d}$, Antoine \\ Manzanera $^{\mathrm{c}}$, Odemir M. Bruno ${ }^{\mathrm{b}, \mathrm{a}}$ \\ ${ }^{a}$ Institute of Mathematics and Computer Science \\ University of São Paulo \\ Avenida Trabalhador São-Carlense, 400, Centro \\ 13566-590 São Carlos, SP, Brazil \\ ${ }^{b}$ São Carlos Institute of Physics \\ University of São Paulo \\ PO Box 369, 13560-970, São Carlos, SP, Brazil \\ ${ }^{c}$ U2IS, ENSTA Paris, Institut Polytechnique de Paris, \\ 828 Boulevard des Maréchaux, 91120 Palaiseau, France \\ ${ }^{d}$ Curso de Engenharia da Computação \\ Programa de Pós-Graduação em Engenharia Elétrica e de Computação \\ Universidade Federal do Ceará (Federal University of Ceara), Campus de Sobral \\ Rua Coronel Estanislau Frota, 563, Centro \\ Sobral, Ceará, CEP: 62010-560, Brasil
}

\begin{abstract}
Dynamic textures (DTs) are pseudo periodic data on a space $\times$ time support, that can represent many natural phenomena captured from video footages. Their modeling and recognition are useful in many applications of computer vision. This paper presents an approach for DT analysis combining a graph-based description from the Complex Network framework, and a learned representation from the Randomized Neural Network (RNN) model. First, a directed space $\times$ time graph modeling with only one parameter (radius) is used to represent both the motion and the appearance of the DT. Then, instead of using classical graph measures as features, the DT descriptor is learned using a RNN, that is trained to predict the gray level of pixels from local topological measures of the graph. The weight vector of the output layer of the RNN forms the descriptor.

Several structures are experimented for the RNNs, resulting in networks with final characteristics of a single hidden layer of 4, 24, or 29 neurons, and input layers 4 or 10 neurons, meaning 6 different RNNs. Experimental results on DT recognition conducted on Dyntex++ and UCLA datasets show a
\end{abstract}


high discriminatory power of our descriptor, providing an accuracy of $99.92 \%$, 98.19\%, $98.94 \%$ and $95.03 \%$ on the UCLA-50, UCLA-9, UCLA-8 and Dyntex++ databases, respectively. These results outperform various literature approaches, particularly for UCLA-50. More significantly, our method is competitive in terms of computational efficiency and descriptor size. It is therefore a good option for real-time dynamic texture segmentation, as illustrated by experiments conducted on videos acquired from a moving boat.

Keywords: Dynamic Texture, Complex Networks, Learned Features, Randomized Neural Networks

\section{Introduction}

Dynamic textures (DT) are visual patterns that vary both spatially and temporally. Therefore, they can represent a wide range of time-varying natural phenomena, such as fire, sea waves, fountains, smoke, among others. The dynamics of the textures are related to the objects or processes present in the videos, that exhibit a certain stationarity with respect to both space and time 1]. In general, they can be characterized by simple and repetitive patterns and non-rigid motion guided by non linear and stochastic dynamics such as swaying branches or bubbling water 2, 3. Over the last years, DTs have received significant attention from the computer vision community, due to their several applications, such as face spoofing detection [4, traffic monitoring [5], crowd behavior analysis [6, fire detection [7, etc.

The basic task of DT recognition consists in classifying a piece of video (i.e. a 3D space $\times$ time volume of data), with respect to meaningful classes such as those mentioned earlier. This task can then be extended to further goals like semantic segmentation of dynamic surfaces, or discriminative detection of objects over non stationary backgrounds. The notorious difficulty of DT recognition comes from the large variability and the diffuse character of natural textures that, unlike objects, cannot - in general - be precisely located in space and time. To these challenges are added those specific to the dynamic nature 
of the problem: the model should represent the different types of movement that can affect the texture: flow, swell, growth, waving, etc. In addition, as a basic step, DT recognition is expected to work in a fast and reactive manner. It must then be able to run in real-time and to adapt itself on line to a changing environment. Motivated by these challenges, a range of approaches has been proposed, that can be divided into the following five categories: (i) filter-based, (ii) motion-based, (iii) discrimination-based, (iv) learning-based and (v) modelbased methods.

The filter-based methods extend still texture analysis techniques to characterize the dynamic textures at different scales in the spatio-temporal domain. In [8, 9], the authors used oriented energy filters to extract the spatio-temporal characteristics of the dynamic textures. Then, Arashloo and Kittler [10] developed a multiresolution binarized statistical image features approach that generates binary code by filtering operations on different regions of the space $\times$ time and in three orthogonal planes. Independent component analysis learns the filters for each orthogonal plane, which generally represents a significant computational cost. Feichtenhofer et al. 11] presented a bag of space $\times$ time energies framework that extracts primitive features from a bank of spatio-temporally steerable filters. In [12] is proposed the spatio-temporal Directional Number transitional Graph (DNG) descriptor. The feature extraction is based on the direction of the temporal flow to compute the structure of the local neighborhood and the transition of the principal directions between frames. Other approaches explore high-order features from Gaussian gradients [13] or use unsupervised 3D filter learning and local binary encoding [14. In the same way, in [15], the authors introduced a novel filtering kernel, named difference of derivative Gaussians, which is based on high-order derivative of a Gaussian kernel. The methods of this category have achieved good results in several databases, however, they are generally limited both in terms of computational complexity and performance.

The motion-based techniques essentially use the kinematic information estimated from frames to describe the dynamic textures. Many techniques use 
the optical flow due to its efficiency in the description of the motion, like [16 that used the global magnitude and direction of the vector field of normal flow. Other approaches are based on the combination of normal flow features and periodicity [17, multi-resolution histogram of the velocity and acceleration fields [18] or rotation and scale invariant features of the image distortions computed using optical flow [19]. More recently, Nguyen et al. [20] presented an operator based on local vector patterns that encode the local motion features from beams of dense trajectories with good results. In 21] the authors developed a method that is based on the time-varying vector field and used singular patterns pooled from the bag-of-keypoint-based coefficients dictionary. Although the methods from this category are efficient for motion description, they often neglect most of the appearance information, which is essential in many problems. Furthermore, the optical flow techniques suppose brightness constancy and local smoothness in the dynamic textures, which can be a very strong constraint [12].

Discrimination-based methods generally use local features such as the Local Binary Patterns (LBP), widely used in image analysis. In fact, most of the methods based on discrimination for dynamic textures are extensions of LBP methods to the space $\times$ time domain. In this sense, Zhao and Pietikäinen [22, 23. proposed the two most popular methods, which have the advantage of simplicity. The Volumetric LBP (VLBP) [23] encodes the local feature by means of 3D neighborhood and uses as feature vector a very large histogram (i.e., 16384 descriptors). Next, the authors proposed the LBP-TOP [22], which applies the LBP operator on three orthogonal planes (two temporal planes and one spatial plane) and combines the three histograms, reducing the size of the feature vector. Recently, other works also have extended the LBP operator such as multiresolution edge-weighted local structure pattern [24], helix local binary pattern [25] and rotation-invariant version [26]. On the other hand, in [27] the authors introduced the LTGH descriptor, which combines LBPs and gray-level co-occurrence matrix (GLCM) on orthogonal planes (TOP). Nguyen et al. 28] proposed the momental directional patterns framework that extends the Local Derivative Pattern operator to improve the capture of directional features. In 
[29, it is proposed the local tetra pattern operator on three orthogonal planes, which computes feature codes based on the central pixel and directions of the neighbors. In general, these methods provide promising performances, however, they have some limitations such as sensitivity to noise 25] and large feature vectors [23, 30].

Following the success of the deep convolutional neural networks (CNN) on image classification, there has recently been a growing interest in learningbased methods for dynamic texture analysis. Qi et al. 31 applied pre-trained CNN to extract mid-level features from the frames. The first and second order statistics from the mid-level features are used to create the feature vector. Later, Arashloo et al. 32 presented a deep multi-scale convolutional network (PCANet-TOP) architecture that learns filters employing the principal component analysis (PCA) on each orthogonal plane. Andrearczyk and Whelan [33] proposed a framework based on applying CNNs on three orthogonal planes. This framework used the AlexNet and GoogleNet models that were trained on spatial frames and temporal slices from the dynamic texture videos. The output of the three CNNs are combined to obtain a feature vector. The approaches of this category are powerful and usually obtain outstanding results in DT classification. However, they have known limitations that can make them unfeasible for many real-world problems, such as their difficulty to be implemented on embedded platforms, and the need for a considerable number of training samples, that can be impossible to get, particularly when online adaptation/learning is needed. Zhao et al. 34 explore two different approaches to learn 3D random features: learning-based Fisher vector and the learning-free binary encoding. In 35] is proposed a DT descriptor, which employs Randomized Neural Networks (RNNs) to learn the local features from three orthogonal planes. The determining interest is that the RNN has a single feed-forward hidden layer and a fast learning algorithm, making the feature extraction extremely efficient. In the model-based category, the methods analyze the DTs through mathematical or physical models. Popular methods of this category are based on linear dynamical systems (LDS). In [1], the estimated parameters 
of the LDS model are used for characterizing the DT. However, the method has limitations such as a poor invariance to rotation, scale and illumination 33 . To overcome the view-invariance limitation, Ravichandran et al. [36] proposed the Bag-of-dynamical Systems (BoS), which uses the LDSs features with nonEuclidean parameters computed from non linear dimensionality reduction and clustering. Later, in [37 the authors extended the method to extract interest points with a dense sampling and used two alternative approaches for forming the code books. Wang and $\mathrm{Hu} 38$ also proposed a bag-of-words approach to encode chaotic features. More recently, methods that use deterministic walkers [39] and Complex Network (CN) theory [40, 3] have been used with success for DT analysis. In particular, the CN-based methods obtained great results due to their flexibility and ability to represent the motion and appearance in the DTs. These methods model the DT as graphs, and extract statistical measures from them. Despite the promising results, we believe that a more robust characterization of the graph can improve the performance compared to the classic statistical measures. Furthermore, for graph modeling, these methods have as a drawback the need to adjust four parameters.

In summary, the main drawbacks present in the existing methods from the literature are: (i) strong emphasis on either appearance or motion, and poor combination of the two aspects; (ii) large feature vectors; (iii) complex and computationally costly algorithms; (iv) many parameters to adjust; and (v) very simple graph measures.

To address these problems, we present in this paper a DT method that extends the approach proposed in [41] for static textures, that is, it combines a graph based description from the Complex Network (CN) framework, and a learned representation from the Randomized Neural Network (RNN) model. Using a small piece of DT data, the RNN can be trained to predict the gray level value of a pixel, from its local topology features, provided by measures on the space $\times$ time graph of the video. The learned weight vector of the output layer of the RNN is then used as descriptor of the video. Thus, we refer to our method as Complex Patterns learned using Randomized Neural Networks 
(CPNN). Our contributions are:

- A more simple directed graph modeling than [3, 40, 42, for dynamic textures based on only one parameter (radius).

- A robust learned representation for graphs using the RNN, instead of using classic statistical measures. The RNN is an extremely compact neural network that has a fast and few-shots learning algorithm, which is a determining advantage with respect to deep neural network approaches.

- A DT descriptor that provides competitive accuracy and processing time compared to many literature methods.

We evaluate this descriptor in recognition task using different classifiers on two popular DT datasets. Then, we illustrate the potential reactivity of the model on a DT segmentation example. This paper is organized as follows: Section 2 presents the related background on CN and RNN. Section 3 explains our approach in detail. Section 4 explains our experiments and shows some results. Section 5 presents and discusses quantitative results on DT recognition, and qualitative results on reactive DT segmentation. Finally, Section 6 presents the conclusions and future works.

\section{Methodologies}

\subsection{Complex Networks}

The complex network research field emerges from the intersection of the areas of graph theory, physics, statistics and computer science, in order to understand and analyze complex systems. Indeed, many systems and data are formed by a set of elements that interact with each other and that can be represented as networks by defining the entities (vertices) and the relationships among them (edges). Some examples of these systems are the internet, the WWW and social networks. In particular, researches in scale-free networks [43], identification of community structure in many networks [4] and small-world [45] networks 
have drawn attention from the scientific community on the study of complex networks, which is a multidisciplinary research field. In computer vision, the flexibility and expressiveness of this framework have been used over the last years for color-texture classification with multi-layer $\mathrm{CN}$ [46] and dynamic texture analysis with diffusion in networks [3].

Formally, a complex network can be defined as a graph $G=(V, E)$, where $V$ is the set of vertices and $E$ the set of edges connecting vertex pairs. In this work, we adopted the term graph to refer to a complex network in order to avoid confusion with the neural network term. The graphs can be directed and weighted, which is when the edge $e_{i j}$ is directed from $i$ to $j$ and has a weight $w_{i j} \in \mathbb{R}$. For description, two important information about the graph can be used: the out-degree and the weighted out-degree or strength. The out-degree of a vertex $i$ counts the number of connections from $i$ :

$$
k_{i}=\left|\left\{v_{j} ; e_{i j} \in E\right\}\right|,
$$

where $|S|$ denotes the cardinality of set $S$. The weighted out-degree $s_{i}$ computes the sum of the weights of all connections from $i$ :

$$
s_{i}=\sum_{e_{i j} \in E} w_{i j}
$$

These measures quantify topological characteristics and different properties of the graph that are useful for the identification of hubs, scale-free property, etc.

\subsection{Randomized Neural Networks}

A well known problem in neural networks is that gradient descent based learning is slow and needs a huge quantity of data, specially when the number of neural weights is large. To tackle this issue, randomized neural networks [47, 48, 49, 50] were proposed. In their simplest version, these networks have a single hidden layer whose weights are random, and an output layer whose weights can be computed using a closed-form solution. 
To mathematically describe the neural network used in this work, let $Z=$ $\left[\overrightarrow{z_{1}}, \overrightarrow{z_{2}}, \ldots, \overrightarrow{z_{N}}\right]$ be a matrix composed of the outputs of the hidden layer, according to the following equation

$$
Z=\phi\left(\left[\overrightarrow{w_{1}}, \overrightarrow{w_{2}}, \ldots, \overrightarrow{w_{Q}}\right]^{T}\left[\overrightarrow{x_{1}}, \overrightarrow{x_{2}}, \ldots, \overrightarrow{x_{N}}\right]\right)
$$

where $T$ denotes transpose operation, $\overrightarrow{x_{i}}=\left[-1, x_{i 1}, x_{i 2}, \ldots, x_{i p}\right]^{T}$ is an input vector $i$ with $p+1$ attributes, $\vec{w}_{q}=\left[w_{q 0}, w_{q 1}, \ldots, w_{q p}\right]^{T}$ is the set of random weights of a determined neuron $q, N$ is the number of feature vectors $\overrightarrow{x_{i}}, Q$ is the number of neuron units of the hidden layer, and $\phi($.$) is a transfer function.$

Next, after adding a constant value -1 to each vector $\overrightarrow{z_{i}}$ for the bias weights of the output layer neurons, the aim of the closed-form training is to find a matrix $M$ that satisfies $D=M Z$, where $D=\left[\overrightarrow{d_{1}}, \overrightarrow{d_{2}}, \ldots, \overrightarrow{d_{N}}\right]$ is a matrix of (ground truth) label vectors, each one corresponding to its respective input $\overrightarrow{x_{i}}$. To compute $M$, it is possible to use the Moore-Penrose pseudo-inverse [51, 52 with the Tikhonov regularization [53, 54, thus resulting in the following equation

$$
M=D Z^{T}\left(Z Z^{T}+\lambda I\right)^{-1}
$$

where $0<\lambda<1$ and $I$ is an identity matrix of size $(Q+1) \times(Q+1)$.

\section{Proposed Approach}

In this section, we describe the CPNN approach, which extends to dynamic textures the static texture characterization approach proposed in [41. In the first step, the dynamic texture video is modeled into two directed graphs: spatial and temporal graphs. Then, information from these graphs are used to train the neural networks and build a signature.

\subsection{Modeling Dynamic Texture in Directed Graphs}

In order to analyze the dynamic textures, it is important to capture features that represent the appearance and motion properties of the video. To this end, 
in the proposed approach, we model the dynamic texture video into two directed graphs: the spatial graph $G_{S}=\left(V_{S}, E_{S}\right)$ that represents the appearance properties and the temporal graph $G_{T}=\left(V_{T}, E_{T}\right)$ that contains the motion characteristics. Each pixel $i=\left(x_{i}, y_{i}, t_{i}\right)$ of the graphs is represented by a vertex $i \in V$, such that $x_{i}$ and $y_{i}$ are the spatial coordinates and $t_{i}$ the temporal index of the pixel $i$.

The two graphs have as main difference the definition of the set of edges. In the spatial graph, in order to characterize appearance, we connect only vertices that are from the same frame. Thus, the set $E_{S}$ is given by the connection of all vertices whose distance is smaller than or equal to a given radius $r$ and whose time coordinates $t_{i}$ and $t_{j}$ are equal (as illustrated in Figure 1(a)),

$$
e_{i j} \in E_{S} \Longleftrightarrow\left(\left(x_{i}-x_{j}\right)^{2}+\left(y_{i}-y_{j}\right)^{2}+\left(t_{i}-t_{j}\right)^{2}\right) \leq r \text { and } t_{i}=t_{j}
$$

On the other hand, in the temporal graph, we focus on the relationships between frames to analyze the motion characteristics. In this way, in the set $E_{T}$, we connect all vertices whose distance is smaller than or equal to $r$ and their time coordinates are different (as exemplified in Figure 1 (b)),

$$
e_{i j} \in E_{T} \Longleftrightarrow\left(\left(x_{i}-x_{j}\right)^{2}+\left(y_{i}-y_{j}\right)^{2}+\left(t_{i}-t_{j}\right)^{2}\right) \leq r \text { and } t_{i} \neq t_{j}
$$

(5)

to the texture patterns. To add this information to the graph topology, we transform it to a directed and weighted graph, as follows: First, for each edge a direction is defined based on the order of gray-level. Specifically, in an edge $e_{i j}$ the vertex $i$ points to the vertex $j$ if $I(i) \leq I(j)$. In addition, a weight $w_{i j}$ is defined from the difference of intensities and distance between the two pixels 
that represent the vertices:

$$
w_{i j}=\left\{\begin{array}{cc}
\frac{|I(i)-I(j)|}{L}, & \text { if } r=1 \\
\frac{\left(\frac{d i s t(i, j)-1}{r-1}\right)+\left(\frac{|I(i)-I(j)|}{L}\right)}{2}, & \text { otherwise. }
\end{array}\right.
$$

where $I(i) \in[0,255]$ is the gray-level of the pixel $i$, dist $(i, j)$ is the Euclidean distance between the pixels $i$ and $j$ and $L$ is the highest gray-level. When the radius is $r=1$ then the weight $w_{i j}$ is the difference of gray levels normalized by the maximum gray-level $L$, producing a value in $[0,1]$. On the other hand, if $r>1$ then the weight $w_{i j}$ is given by the average of the distance between the pixels normalized by the maximum distance $r$, and the normalized gray scale difference, which also provides a value in $[0,1]$. Thus, this weight function includes information about the pixel neighborhood and the difference of graylevel in order to balance the importance between geometric information and color in the texture representation [40].

\subsection{Proposed Signature}

In this approach, we train the randomized neural network with topological characteristics from graphs that model the videos of dynamic textures. For training the RNNs, the out-degree $k_{i}$ and the weighted out-degree $s_{i}$ of each vertex from the modeled graph compose the input matrices. The learned weights from the output layer form the feature vector for dynamic texture representation. The main steps of the proposed method are summarized in the flowchart diagram in Figure 3.

In order to create the matrix of input vectors for randomized neural network, we use the evolution of the graph for different values of modeling parameter $r$. Therefore, for each vertex $i$ of the graph an input vector and its corresponding output label are defined as follows: the out-degree values of the vertex for different radii $\left\{r_{1}, r_{2}, \ldots, r_{R}\right\}$ are considered as the input vector $\vec{x}_{i}=\left[k_{i}^{1}, k_{i}^{2}, k_{i}^{3}, \ldots, k_{i}^{R}\right]$, where $R$ is the maximum radius value. The output label is simply the gray-level $d_{i}=I(i)$. A matrix of input vectors $X_{(k)}$ for the out-degree and a vector of output labels $D$ is obtained considering all vertices 


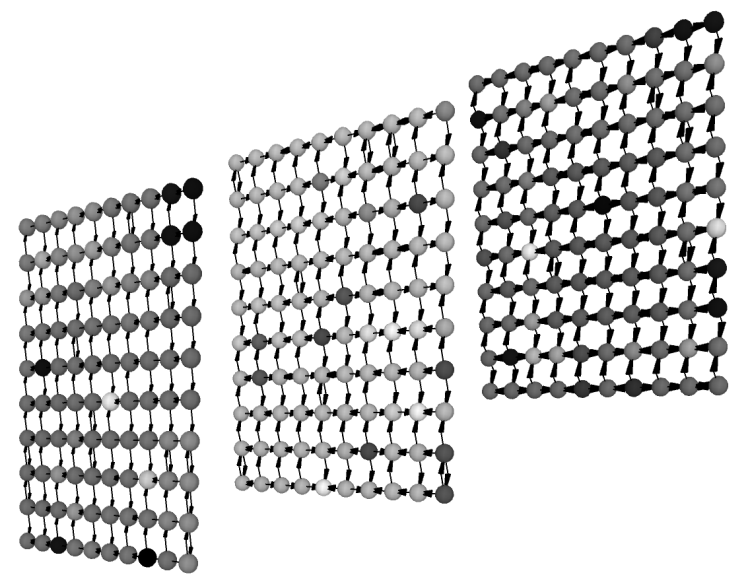

(a) Spatial graph

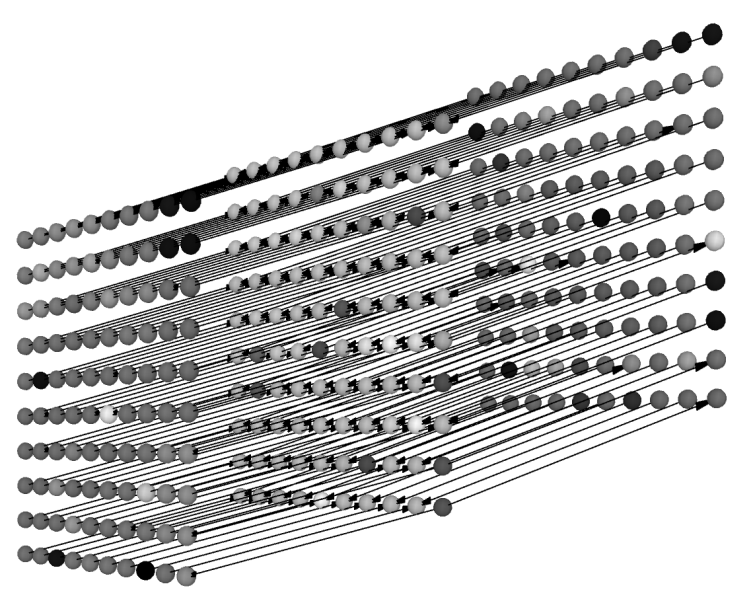

(b) Temporal graph

Figure 1: Modeling of a three frame video in a (a) spatial graph and a (b) temporal graph (using $r=1$ ).

of the graph. The number of columns of $X_{(k)}$ (and the size of vector $D$ ) is then the number of samples $N$ which corresponds to all the pixels, but could also be any sub-sample of the video. In this way, it is possible to statistically analyze the topology evolution of the vertices that represent the pixels with different gray-levels. Figure 2(a) illustrates the step for obtaining the matrices of input vector and label from a spatial graph. In addition to the matrix $X_{(k)}$, we also 
build the matrix of input vectors for the weighted out-degree $X_{(s)}$.

(a)

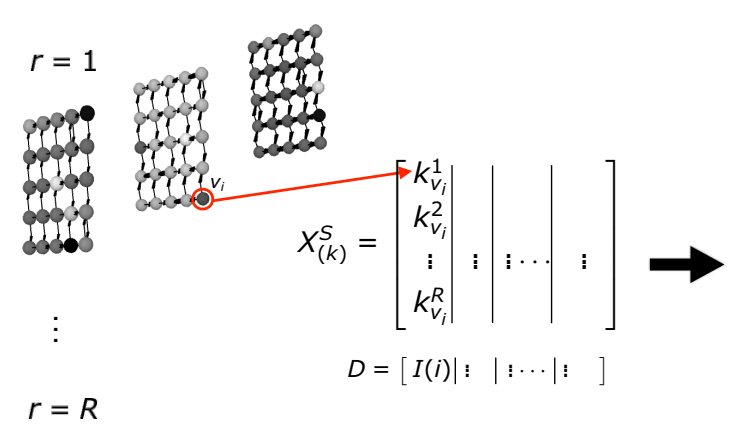

(b)

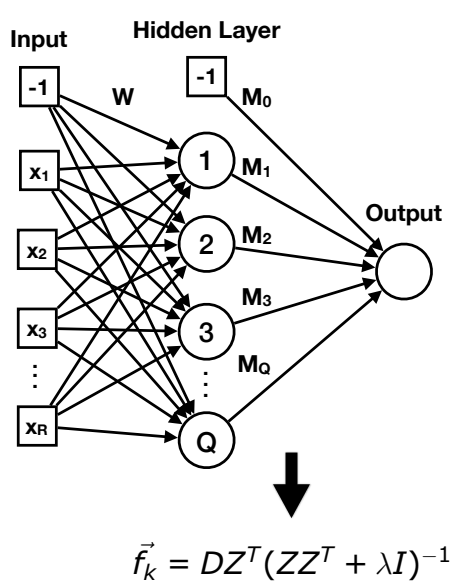

Figure 2: Construction of the output vector and label from a spatial graph by modeling dynamic textures with different values of $r$ and using the out-degree $k_{i}$.

In the next step, the weights of the matrix $W$ of the input layer of the randomized neural network are generated randomly. However, in the methods for dynamic texture analysis it is important that the feature vector be always the same for a given sample. In this sense, in order to always obtain the same weight values, we use the Linear Congruent Generator (LCG) [55, 56] with fixed parameters to generate the uniform pseudo random numbers for the matrix $W$,

$$
V(n+1)=(a * V(n)+b) \bmod c
$$

where $V$ is the random sequence of length $E=Q *(p+1)$ and started by $V(1)=E+1$. The values of $a, b$ and $c$ are parameters defined as $a=E+2$, $b=E+3$ and $c=E^{2}$ (these values were adopted in [57). Thus, the matrix $W$ is composed of the vector $V$ divided into $Q$ segments of values $p+1$. The values of the matrix $W$ and $X$ (each row) are normalized using standard score (zero mean and unit variance).

The feature vector that represents the dynamic textures is built based on matrix $M$, which becomes here a vector $\vec{f}$ (since the output labels are scalar), 
which is computed as: $\vec{f}=D Z^{T}\left(Z Z^{T}+\lambda I\right)^{-1}$, such that $\lambda=10^{-3}$ (Figure $2(\mathrm{~b}))$ and the length of $\vec{f}$ is $Q+1$ because of the bias value. To characterize appearance and movement of dynamic textures, we propose to use the spatial graph and temporal graph as inputs to train the randomized neural network. Therefore, firstly, two randomized neural networks are trained, each one with a different matrix of input vectors $X_{(k)}^{S}$ and $X_{(s)}^{S}$ extracted from the spatial graphs. From these trained randomized neural networks, we obtain two vectors $\vec{f}_{k}^{S}$ and $\vec{f}_{s}^{S}$. For the temporal graph, the same procedure is performed and two vectors are obtained ${\overrightarrow{f_{k}}}^{T}$ and $\vec{f}_{s}^{T}$ using the matrices $X_{(k)}^{T}$ and $X_{(s)}^{T}$, respectively. In this way, to represent the appearance and motion of the dynamic texture, the following concatenation is proposed:

$$
\vec{\Upsilon}(Q)_{R}=\left[{\overrightarrow{f_{k}}}^{S},{\overrightarrow{f_{s}}}^{S},{\overrightarrow{f_{k}}}^{T},{\overrightarrow{f_{s}}}^{T}\right]
$$

where $Q$ is the number of hidden layer neurons and $R$ is the maximum radius of graph modeling, i.e., the number of radii used to construct the matrix $X$. Figure 3 illustrates the steps to obtain the vector $\vec{\Upsilon}(Q)_{R}$, which is built using a single value of $Q$ and $R$. These two parameters influence the training of the neural network and, therefore, different characteristics are learned for different parameter values. Thus, the vector $\vec{\Psi}(Q)_{R_{1}, R_{2}}$ that concatenates the vectors $\vec{\Upsilon}(Q)_{R}$ for different values of $R$ is used:

$$
\vec{\Psi}(Q)_{R_{1}, R_{2}}=\left[\vec{\Upsilon}(Q)_{R_{1}}, \vec{\Upsilon}(Q)_{R_{2}}\right]
$$

Finally, we propose a feature vector $\vec{\Theta}(R)_{\left(Q_{1}, Q_{2}, Q_{m}\right)}$ that combines the vector $\vec{\Psi}(Q)_{R_{1}, R_{2}}$ for different numbers of neurons:

$$
\vec{\Theta}_{Q_{1}, Q_{2}, \ldots, Q_{m}}=\left[\vec{\Psi}\left(Q_{1}\right)_{R_{1}, R_{2}}, \vec{\Psi}\left(Q_{2}\right)_{R_{1}, R_{2}}, \ldots, \vec{\Psi}\left(Q_{m}\right)_{R_{1}, R_{2}}\right]
$$

The overall algorithm of the proposed method is described in Algorithm 1 . 


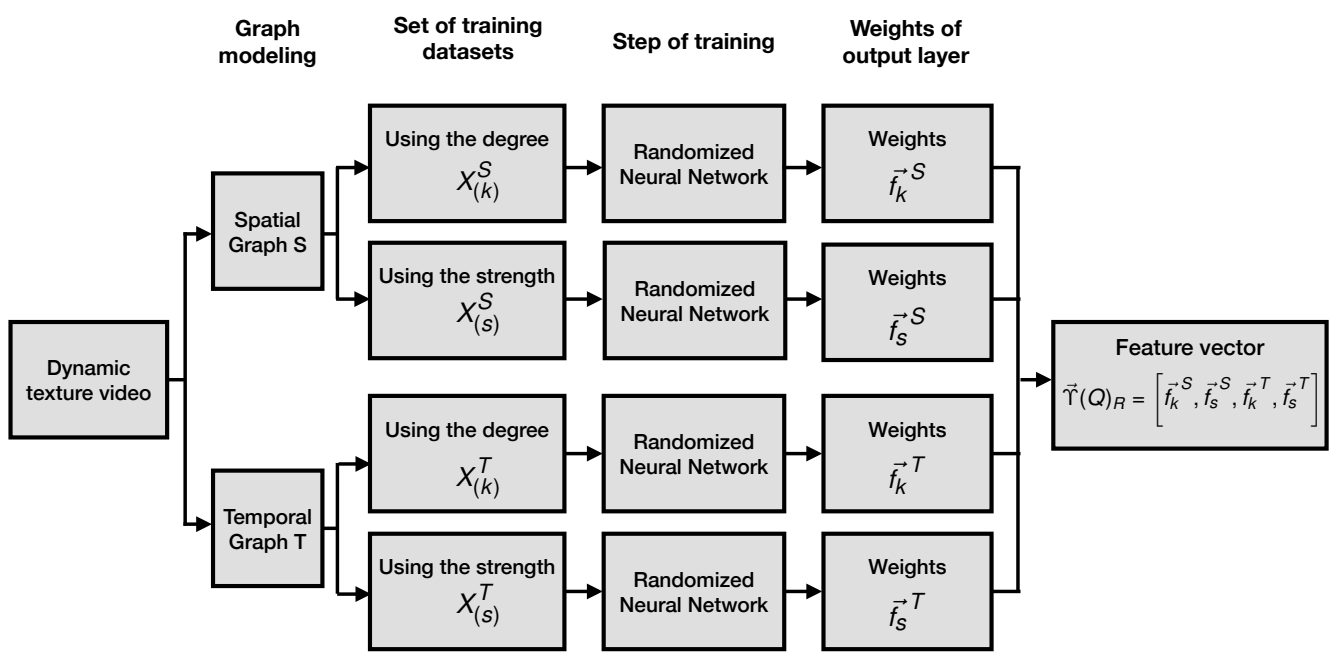

Figure 3: Flowchart diagram of the proposed method. 


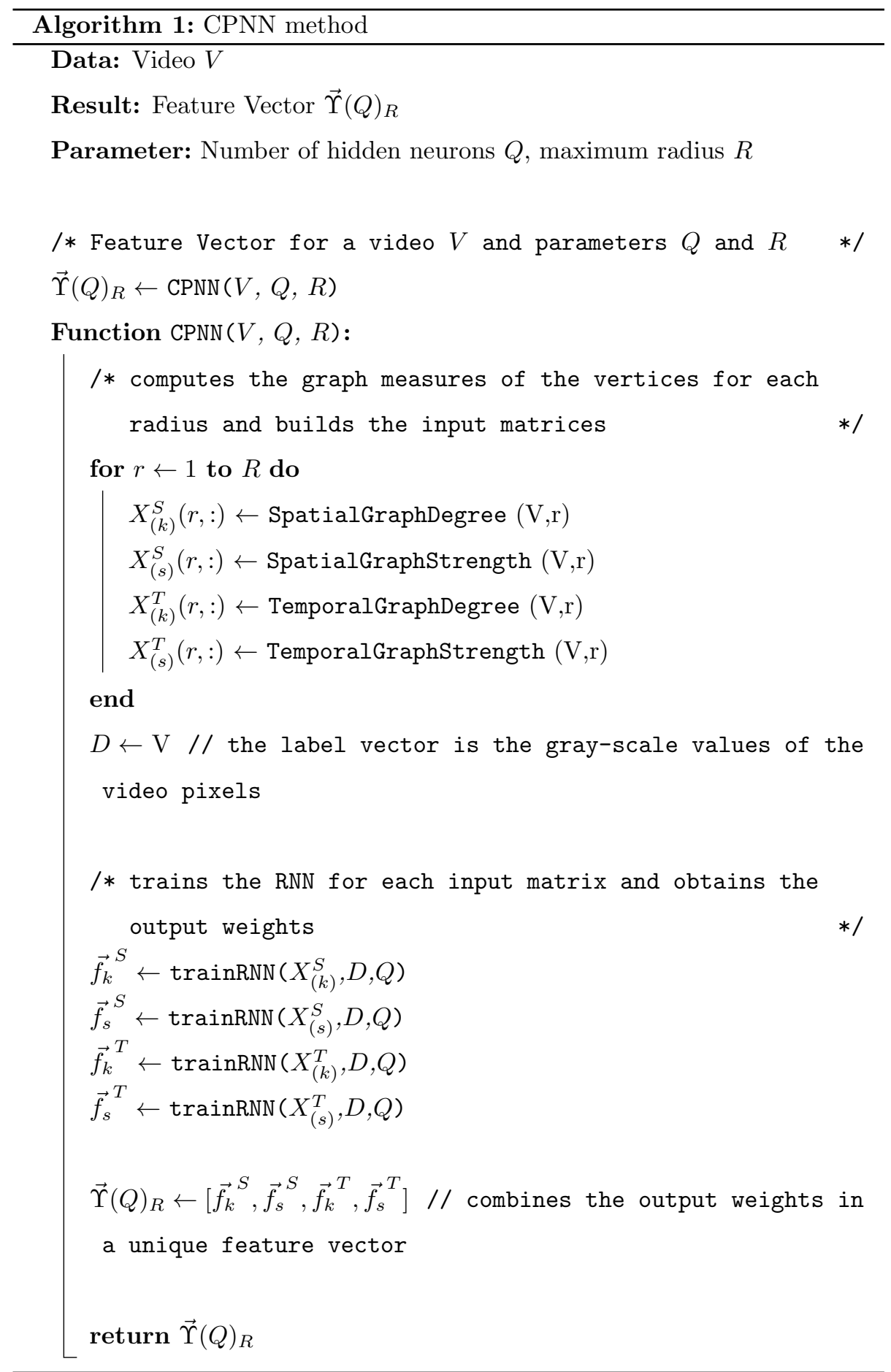




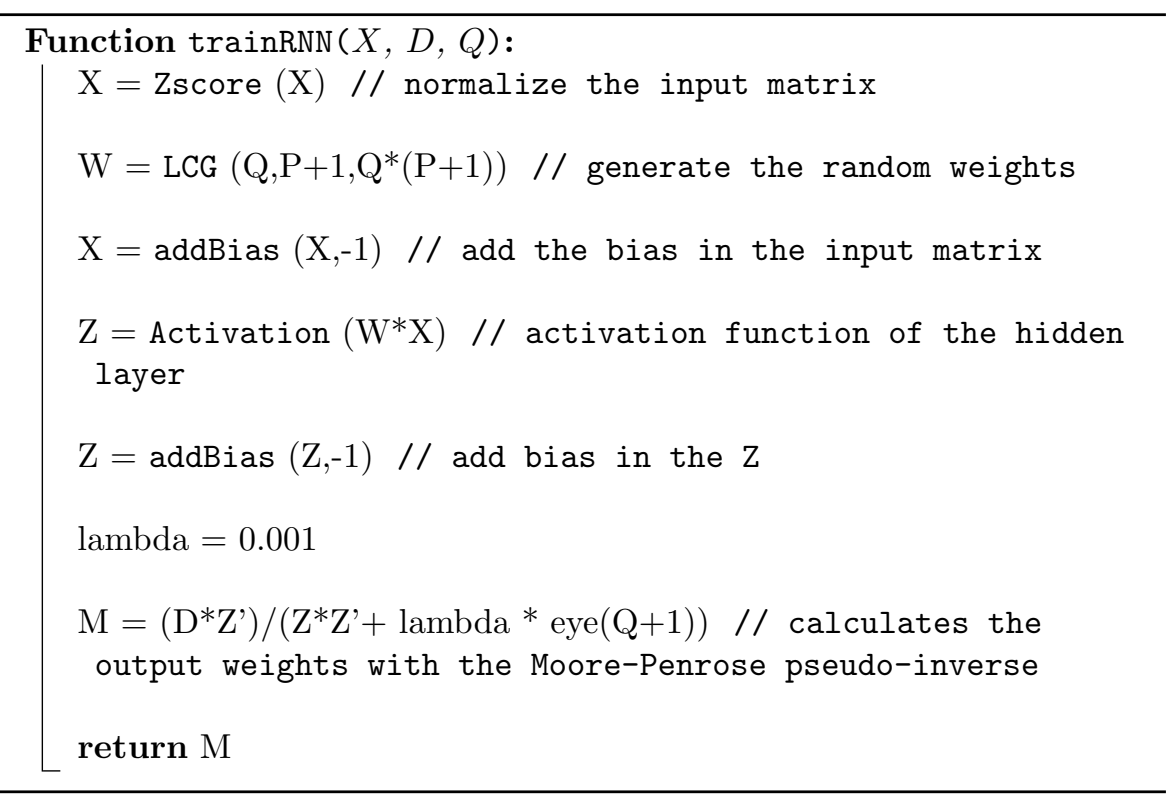

\section{Validation Setup}

To evaluate our method, two benchmark databases were used. They are:

- Dyntex++ [58]: this database, which is a compilation of the Dyntex databases [59], has 3600 videos divided into 36 classes, 100 videos per class, each one of size $50 \times 50 \times 50$. Figure 4 (a) shows examples of the first frame of samples from Dyntex ++ .

- UCLA-50 60]: this database is composed of 50 classes, 4 videos per class, each one of size $75 \times 48 \times 48$. Also, two variations from the UCLA-50, both proposed in 36, were used in our experiments. The first (UCLA-9) combines videos taken from different viewpoints and groups them into 9 classes: smoke (4 samples), flowers (12), boiling water (8), sea (12), fire (8), water (12), fountains (20), waterfall (16) and plants (108). The second (UCLA-8) discards the class "plants" because it has a large set of samples when compared to the other classes. The first frame of some samples from the UCLA database is shown in Figure 4 (b). 
In the validation procedure, we used the 1-NN classifier (we used a implementation from Weka [61]) in order to compare our results to other descriptors available in the literature. As evaluation protocol, we employed the procedure adopted in 36, 58, 25] for the UCLA-50, UCLA-9, and UCLA-8; and adopted in [40] for the Dyntex++. Thus, we used 4-fold and 10-fold cross-validation for the UCLA-50 and Dyntex++, respectively. In this case, we use 10 trials for a statistically more reliable result. On the UCLA-9 and UCLA-8 databases, we used hold-out (50\% of samples for training and the remainder for test). Average accuracy and standard deviation of 20 trials were used to quantify the performance of our method. The graph modeling step of the proposed method was programmed in the $\mathrm{C}$ language, while for training and feature extraction with the RNN was used the Matlab 9.2 software. The trained weights of the output layer (i.e., the feature vectors) computed by the proposed method in all databases are available in GitHub 1

\section{Results and Discussions}

\subsection{Dynamic Texture Classification}

Firstly, we perform a parametric analysis of our approach. For this, a feature vector is extracted of each sample from UCLA-50 and Dyntex ++ databases using different values of $Q$ and $R$. Figure 5 summarizes the average accuracy for the UCLA-50 and Dyntex++ databases using the feature vector $\vec{\Psi}(Q)_{R_{1}, R_{2}}$ with $Q=4$ and different values and combinations of $R$. In the plot, the rows represent the values of $R_{1}$ and the columns the values of $R_{2}$. The main diagonal corresponds to a single value of $R$ used to build the feature vector. In this last case, the highest average accuracy, 94.51\%, is obtained using $R=4$. On the other hand, when using two values of $R$ combined, the highest average accuracy is achieved using the combination $\left(R_{1}, R_{2}\right)=(4,10)$. This indicates that the combination of local (small radius) and regional (large radius) information from

1 https://github.com/lucascorreiaribas/CPNN 


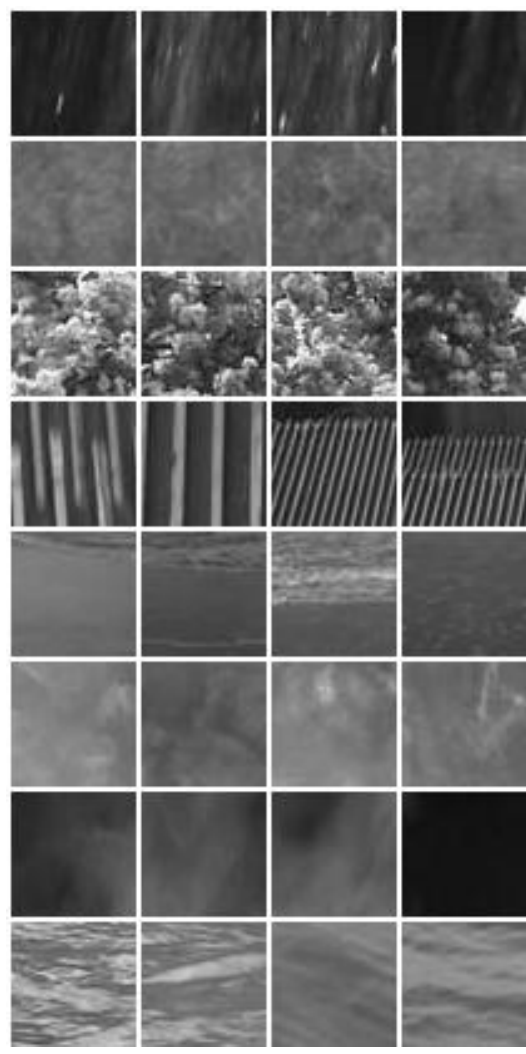

(a) Dyntex++

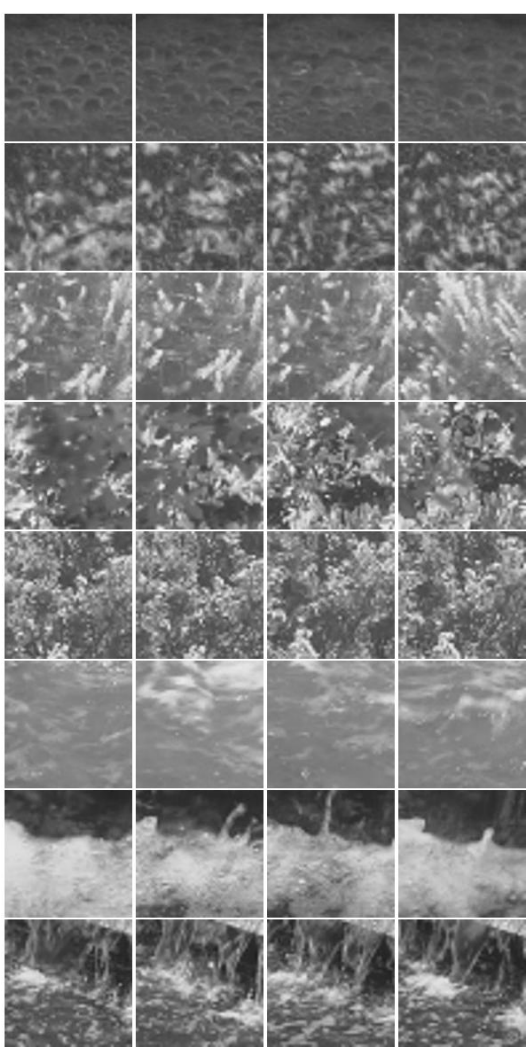

(b) UCLA

Figure 4: Initial frames of dynamic texture samples from the databases. Each row represents a class. 


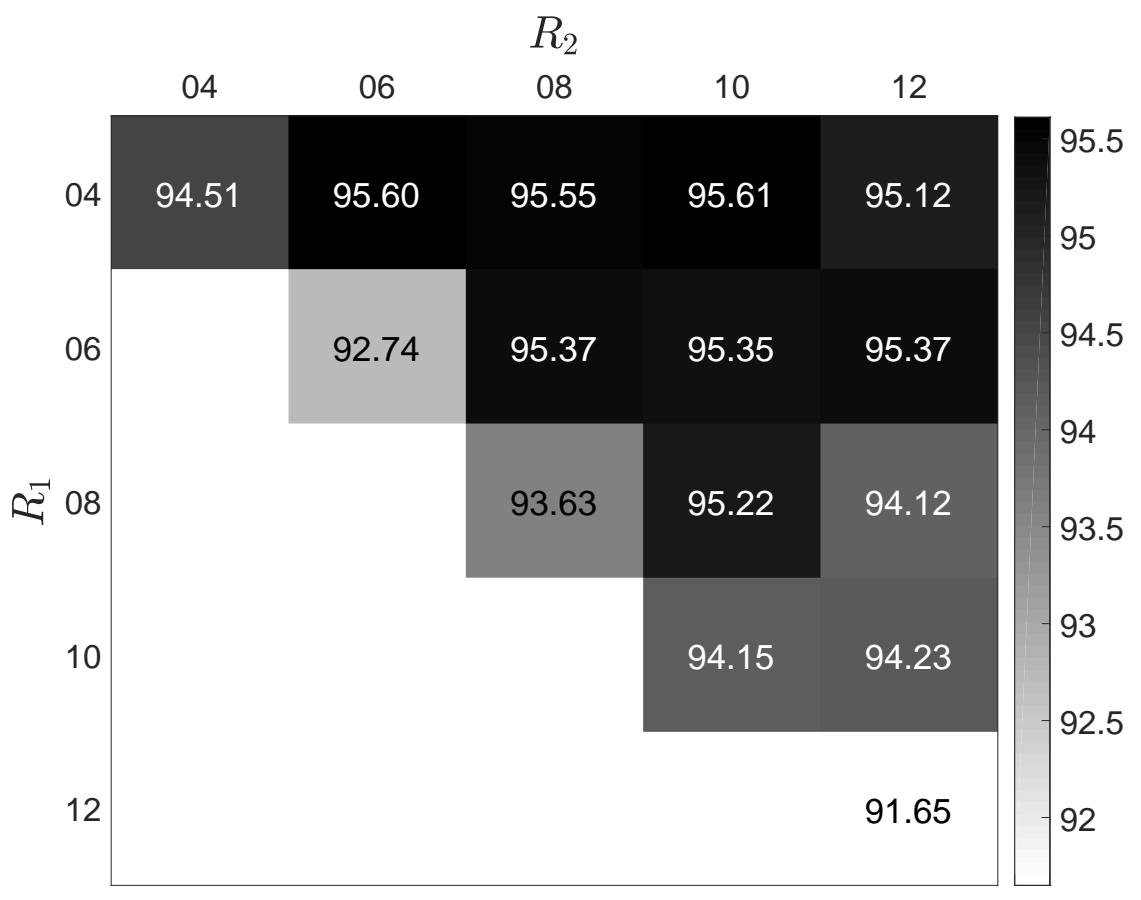

Figure 5: Accuracy for different values and combination of one or two radii, averaged on the datasets UCLA-50 and Dyntex++.

graphs is more discriminative.

Figure 6 presents the accuracies using the feature vector $\vec{\Theta}_{Q_{1}, Q_{2}, \ldots, Q_{m}}$ with one and two values of $Q$ on the UCLA-50 and Dyntex++ databases. It can be seen that, on the UCLA-50 database, the higher accuracies are obtained using large values of $Q$ (all combinations with $Q=29$ ), while small values of $Q$ provide better accuracies on Dyntex ++ database. In a second experiment, we test the feature vector $\vec{\Theta}_{Q_{1}, Q_{2}, \ldots, Q_{m}}$ combining three different values of $Q$, as it can be seen in Table 1. Note that, as we increase the values of $Q$ in the combinations, the accuracy tends to stabilize and then decrease, meanwhile the size of the feature vector is increased. In this sense, the combination $\{4,24,29\}$ can be considered as a good trade-off between the size of feature vectors and accuracy 
Table 1: Accuracy for the feature vector $\vec{\Theta}_{Q_{1}, Q_{2}, \ldots, Q_{m}}$ using three value combinations for $Q$.

\begin{tabular}{cccc}
\hline$Q$ & \# of features & UCLA & Dyntex++ \\
\hline$\{04,09,14\}$ & 240 & $99.10( \pm 1.21)$ & $95.06( \pm 1.04)$ \\
$\{04,09,19\}$ & 280 & $99.10( \pm 1.02)$ & $95.08( \pm 1.14)$ \\
$\{04,09,24\}$ & 320 & $99.50( \pm 0.89)$ & $95.19( \pm 1.10)$ \\
$\{04,09,29\}$ & 360 & $99.50( \pm 0.89)$ & $94.71( \pm 1.21)$ \\
$\{04,14,19\}$ & 320 & $99.30( \pm 0.98)$ & $95.38( \pm 0.97)$ \\
$\{04,14,24\}$ & 360 & $99.40( \pm 0.94)$ & $95.32( \pm 1.09)$ \\
$\{04,14,29\}$ & 400 & $99.60( \pm 0.82)$ & $95.05( \pm 1.16)$ \\
$\{04,19,24\}$ & 400 & $99.10( \pm 1.21)$ & $95.10( \pm 1.10)$ \\
$\{04,19,29\}$ & 440 & $99.50( \pm 0.89)$ & $94.86( \pm 1.18)$ \\
$\{04,24,29\}$ & 480 & $99.92( \pm 0.37)$ & $95.03( \pm 1.27)$ \\
$\{09,14,19\}$ & 360 & $99.10( \pm 1.02)$ & $95.18( \pm 0.91)$ \\
$\{09,14,24\}$ & 400 & $99.50( \pm 0.89)$ & $95.18( \pm 1.04)$ \\
$\{09,14,29\}$ & 440 & $99.50( \pm 0.89)$ & $94.90( \pm 1.10)$ \\
$\{09,19,24\}$ & 440 & $98.80( \pm 1.36)$ & $94.81( \pm 1.15)$ \\
$\{09,19,29\}$ & 480 & $99.10( \pm 1.02)$ & $94.69( \pm 1.21)$ \\
$\{09,24,29\}$ & 520 & $99.30( \pm 0.98)$ & $94.87( \pm 1.12)$ \\
$\{14,19,24\}$ & 480 & $98.80( \pm 1.36)$ & $94.94( \pm 1.02)$ \\
$\{14,19,29\}$ & 520 & $99.10( \pm 1.02)$ & $94.72( \pm 1.19)$ \\
$\{14,24,29\}$ & 560 & $99.40( \pm 0.94)$ & $94.72( \pm 1.01)$ \\
$\{19,24,29\}$ & 600 & $99.00( \pm 1.21)$ & $94.24( \pm 1.11)$ \\
\hline
\end{tabular}

on both databases. However, it is important to emphasize that the method obtained good results for a large number of parameter values, demonstrating robustness to parameter changes. For example, the combination $\{4,14\}$ produces 160 features, and the accuracies are $99.60 \%$ and $95.31 \%$ on the UCLA-50 and Dyntex++ databases, respectively.

We also compare our experimental results in the classification task to the results obtained by other existing methods. Table 2 presents the accuracy obtained for different methods on UCLA-50 database. In these experiments, the results indicate that the proposed method achieved the highest accuracy, which is followed by the CNN-GoogLeNet [33] approach with an accuracy of $99.50 \%$. The next accuracy of $97.15 \%$ was obtained by the Randomized Neural Network based signature (RNN-DT) 35] method.

A statistical hypothesis test was performed to evaluate the significance of the difference in performance between the proposed method and the compared 


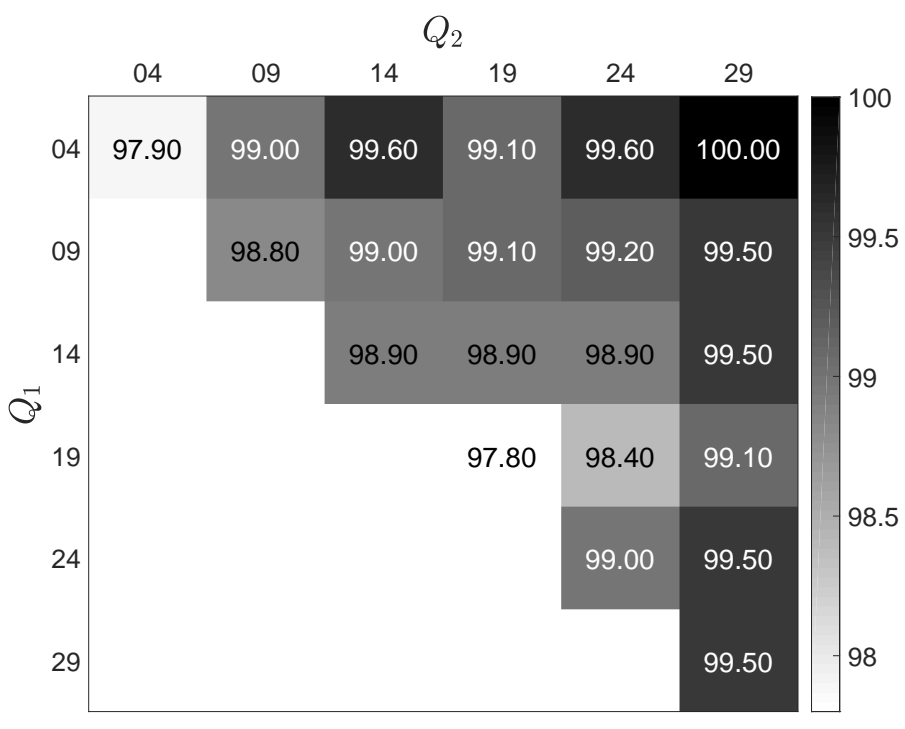

(a) UCLA-50

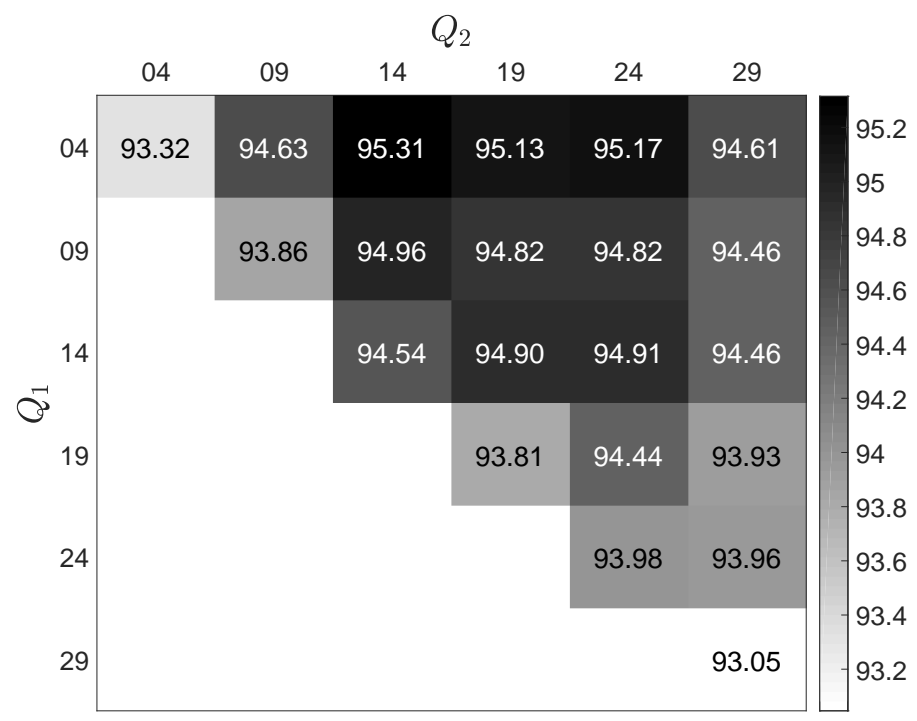

(b) Dyntex++

Figure 6: Classification results for the feature vector $\vec{\Theta}_{Q_{1}, Q_{2}, \ldots, Q_{m}}$ with one single $(m=1)$ or two different $(m=2)$ values of $Q$ on the Dyntex++ and UCLA-50 databases. The diagonal cells correspond to single values of $Q$, the others to combinations of two distinct values of $Q$. 
methods on the UCLA-50 database. For this purpose, we employ the pairedsample t-test and Wilcoxon signed-rank test 62] from the Matlab implementation with a significance level equal to $\alpha=0.05$. To perform this test, the accuracy of the proposed method, Diffusion, DPSWNet, and RNN-DT methods were computed as the average of 20 trials. Thus, the null hypothesis is that the performance of the proposed method is equal to the compared methods, while in the alternative hypothesis the proposed method is statistically superior to the others. When our hypothesis is tested in the comparison with the Diffusion method, the $p$-value is $p=5.687 \mathrm{e}-19$ and $p_{w}=1.255 \mathrm{e}-5$ to the t-test and Wilcoxon test, respectively. Since $p$ and $p_{w}<\alpha$ the null-hypothesis can be rejected in favor of alternative hypothesis, confirming that the performance (mean and median accuracy for t-test and Wilcoxon test, respectively) of the proposed method is statistically superior to the Diffusion method in UCLA-50 database. The $p$-values for the comparison with the RNN-DT ( $p=1.844 \mathrm{e}-17$ and $\left.p_{w}=3.094 \mathrm{e}-5\right)$ and DPSWNet $\left(p=6.683 \mathrm{e}-20\right.$ and $\left.p_{w}=1.614 \mathrm{e}-5\right)$ methods are also much lower than $\alpha$ in the two tests, so we can reject the null-hypothesis. Regarding the GoogLenet method, the result of the statistical test must be taken with caution because we use the result of the original paper. Therefore, our hypothesis is that the proposed method improved the result in relation to the GoogleLenet in $0.25 \%$, so the null-hypothesis is the negation of this hypothesis. Based on the t-test and Wilcoxon test, the $p$-values are $p=0.0002$ and $p_{w}=0.00093$, respectively. These values reject the null-hypothesis and indicate the superiority of the proposed method.

Table 3 provides a comparison of literature methods on the UCLA- 8 and UCLA-9 databases, which consider videos taken from different viewpoints in the same class. On the UCLA- 8 database, the proposed method obtained the second-highest accuracy (98.94\%), which is very similar to the highest accuracy achieved by the CNN-GoogLeNet (99.02\%). On the other hand, on the UCLA-9 database, the DPSWNet 42 method achieved the highest accuracy $(99.10 \%)$ while the proposed method obtained $98.19 \%$. Table 4 presents the comparison results for the Dyntex++ database. On this database, our pro- 
Table 2: Comparison in the 50-class UCLA database (1-NN classifier and 4-fold cross validation). For the methods with '*', the results are taken from [25, 24] and [0], the results with '+' are obtained from the original paper, while the others were generated.

\begin{tabular}{|c|c|}
\hline Descriptor & $\mathrm{ACC}(\%)$ \\
\hline KDT-MD* 63 & 89.50 \\
\hline DFS* 2 & 89.50 \\
\hline 3D-OTF* 64 & 87.10 \\
\hline CVLBP $^{*} 30$ & 93.00 \\
\hline $\mathrm{HLBP}^{*}$ [25] & 95.00 \\
\hline MEWLSP* 24 $^{*}$ & 96.50 \\
\hline LBP-TOP $[22]^{*}$ & 94.50 \\
\hline VLBP* 23 & 89.5 \\
\hline DPSW 39 & $94.60( \pm 1.98)$ \\
\hline CNN-GoogLeNet $^{+} 33$ & 99.50 \\
\hline CNDT [40] & $94.62( \pm 1.04)$ \\
\hline Diffusion $^{+} 3$ & $98.50( \pm 3.37)$ \\
\hline DPSWNet $^{+} 42$ & $98.00( \pm 3.50)$ \\
\hline $\mathrm{RNN} \mathrm{DT}^{+} 35$ & $97.05( \pm 1.87)$ \\
\hline CPNN & $99.92( \pm 0.37)$ \\
\hline
\end{tabular}

posed method was surpassed by the Local Binary Patterns (LBP-TOP) [22], RNN-DT 35 and RI-VLBP 23. In this way, we can affirm that our result is not statistically superior to the others in these two databases. However, it is important to emphasize some aspects of the methods. CNNs are currently among the most powerful approaches in image analysis and have high computational cost. Also, they require a large number of samples for training, which are drawbacks compared to our method. The RNN-DT is a state-of-the-art method that shares with the CPNN the same fundamental core of using neural weights as descriptors. Furthermore, our proposed approach produces a smaller feature vector (480 descriptors but the combination $\{4,14\}$ produces 160 descriptors with competitive accuracies) when compared to the LBP-TOP (768 descriptors) and RI-VLBP (16384 descriptors), MBSIF (6 144 descriptors) and MEWLSP (1536 descriptors). The feature vector size can be crucial in some applications which require low computational cost to classify the DT and memory consumption.

We also compared the results of the proposed method with other approaches in the literature that are more similar to ours. In particular, the RNN-DT 
method uses the same neural network architecture considered in our work, but in a three orthogonal planes scheme to train the model, without the graph modeling information. The RNN-DT method obtained a slightly higher accuracy in the 9-class UCLA and Dyntex++ databases, while the proposed approach improves the accuracy in $2.95 \%$ and $1.2 \%$ when compared with the RNN-DT method on the 50-class and 8-class UCLA databases, respectively. On the other hand, the CNDT and DPSWNet methods employed different ways to model the dynamic texture in graphs with topological statistical measures as descriptors. In relation to these methods, with the exception of the 9-class UCLA database, the proposed method obtained higher accuracies in all databases. These results indicate that the combination of the graph-based description and learned representation from RNNs can improve the characterization of the dynamic texture.

Table 3: Comparison of the proposed method with other dynamic texture methods in the 9-class and 8-class UCLA databases (1-NN classifier and half of the samples for training and the remainder for testing). The results with '*' are taken from 25] and 24, the results with '+' are obtained from the original paper, while the others were generated.

\begin{tabular}{|c|c|c|}
\hline \multirow[t]{2}{*}{ Descriptor } & \multicolumn{2}{|c|}{$\mathrm{ACC}(\%)$} \\
\hline & 9-class UCLA & 8-class UCLA \\
\hline 3D-OTF* 64 & 96.32 & 95.80 \\
\hline CVLBP $^{*} 30$ & 96.90 & 95.65 \\
\hline $\mathrm{HLBP}^{*}[25$ & 98.35 & 97.50 \\
\hline MEWLSP* 24 & 98.55 & 98.04 \\
\hline MBSIF* [10] & 98.75 & 97.80 \\
\hline High level feature* 65 & 92.67 & 85.65 \\
\hline DNGP* 12 & 98.10 & 97.00 \\
\hline WMFS* 66 & 96.95 & 97.18 \\
\hline Chaotic vector* 38 & 85.10 & 85.00 \\
\hline VLBP* 23] & 96.30 & 91.96 \\
\hline LBP-TOP* 22 & 96.00 & 93.67 \\
\hline CNN-GoogLeNet $^{+} 33$ & 98.35 & 99.02 \\
\hline DPSW [39] & $96.33( \pm 2.46)$ & $93.41( \pm 6.01)$ \\
\hline CNDT [40] & $95.61( \pm 2.72)$ & $94.32( \pm 4.18)$ \\
\hline DPSWNet $^{+} 42$ & $99.10( \pm 0.86)$ & $96.55( \pm 7.13)$ \\
\hline Diffusion $^{+}$3] & $97.80( \pm 1.53)$ & $96.22( \pm 4.80)$ \\
\hline 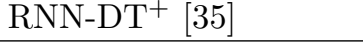 & $98.54( \pm 1.56)$ & $97.74( \pm 2.99)$ \\
\hline CPNN & $98.19( \pm 2.27)$ & $98.94( \pm 1.42)$ \\
\hline
\end{tabular}

In addition to 1-NN, we also consider other classifiers to evaluate the poten- 
Table 4: Comparison of the proposed method and others in the Dyntex ++ database (1-NN classifier and 10-fold cross validation). The results with '*' are obtained from [35, the results with '+' are taken from the original paper, while the others were generated.

\begin{tabular}{|c|c|}
\hline Descriptor & $\mathrm{ACC}(\%)$ \\
\hline VLBP $[23]^{*}$ & $96.14( \pm 0.77)$ \\
\hline LBP-TOP [22]* & $97.72( \pm 0.43)$ \\
\hline DPSW [39] ${ }^{*}$ & $91.39( \pm 1.29)$ \\
\hline CNDT [40] ${ }^{*}$ & $83.86( \pm 1.40)$ \\
\hline${ }_{\text {DPSWNet }}[42]^{+}$ & $93.50( \pm 1.27)$ \\
\hline Diffusion $[3]^{+}$ & $93.80( \pm 1.08)$ \\
\hline RNN-DT [35] ${ }^{+}$ & $96.51( \pm 0.94)$ \\
\hline $\mathrm{CPNN}$ & $95.03( \pm 1.27)$ \\
\hline
\end{tabular}

tial of the methods. The classifiers are: Random Forest 67, Deep Random Vector Functional Link - D-RVFL [68] and Linear Discriminant Analysis - LDA [69]. We consider the dynamic texture descriptors with the features and source codes available. Table 5 shows the accuracies obtained on the UCLA-50, UCLA-9 and UCLA-8 databases. In the table, the rows represent the different DT descriptors, while the columns represent the different classifiers evaluated on each database. On the UCLA-50 database, the proposed method achieved the highest accuracy in all classifiers, which are very similar to the RNN-DT and DPSWNet methods. On the other hand, on the UCLA-9 and UCLA-8 databases, the RNN-DT method had a better performance, except for the UCLA-9 using the Random Forest and D-RVFL classifiers, where our method had a slightly higher accuracy. In particular, we can observe that on UCLA-50 database and using the D-RVFL classifier, our descriptor CPNN achieved a good performance compared to other descriptors, such as Diffusion, RI-VLBP, and DPSW methods. The core of the CPNN method is learning features from randomized neural networks, which can be viewed as a variant of the RVFL network. This can explain the good performance of these methods since their features are the weights of the output layer. This argument motivates the investigation for extending our method to other neural network architectures with more layers, although this may penalize the computational competitiveness of the method. On the Dyntex ++ database, the proposed method also obtained the highest accuracy for the D-RVFL classifier, 
Table 5: Accuracy obtained on the UCLA databases by different dynamic texture descriptors (represented in the rows) using the Random Forest, D-RVFL and LDA classifiers.

\begin{tabular}{l|ccc|ccc|ccc}
\hline \multirow{2}{*}{ Descriptor } & \multicolumn{3}{|c|}{ Random Forest } & \multicolumn{3}{c|}{ D-RVFL } \\
& UCLA-50 & UCLA-9 & UCLA-8 & UCLA-50 & UCLA-9 & UCLA-8 & UCLA-50 & UCDA \\
\hline VLBP & $80.37(1.49)$ & $86.79(4.03)$ & $82.84(6.97)$ & $81.10(1.10)$ & $73.38(4.37)$ & $56.48(4.79)$ & $72.87(1.11$ & $86.32(3.18)$ & $85.00(6.32)$ \\
DPSWNet & $97.30(0.54)$ & $91.53(4.03)$ & $93.07(4.27)$ & $94.15(0.97)$ & $86.84(3.05)$ & $65.45(2.82)$ & $98.37(0.48)$ & $94.90(2.14)$ & $92.50(6.22)$ \\
DPSW & $95.75(1.32)$ & $92.24(3.21)$ & $92.50(4.78)$ & $83.45(2.11)$ & $84.18(3.33)$ & $60.22(7.35)$ & $88.12(1.44)$ & $94.13(4.09)$ & $88.97(6.09)$ \\
CNDT & $94.37(0.25)$ & $92.04(3.75)$ & $90.00(6.93)$ & $91.75(0.87)$ & $87.65(3.03)$ & $63.29(4.96)$ & $97.25(0.29)$ & $92.75(4.73)$ & $92.16(5.59)$ \\
Diffusion & $97.00(0.71)$ & $93.32(2.86)$ & $88.52(6.66)$ & $52.75(4.72)$ & $85.25(2.23)$ & $62.95(3.84)$ & $97.87(0.85)$ & $94.54(3.06)$ & $95.34(4.51)$ \\
RNN-DT & $98.90(0.32)$ & $92.04(2.35)$ & $94.32(3.87)$ & $94.25(0.50)$ & $88.01(2.45)$ & $66.93(1.72)$ & $99.50(0.41)$ & $97.19(2.43)$ & $98.07(3.77)$ \\
CPNN & $99.05(0.44)$ & $93.62(3.32)$ & $90.68(4.66)$ & $96.75(0.87)$ & $88.11(2.67)$ & $64.43(3.63)$ & $99.87(0.25)$ & $96.38(3.71)$ & $94.89(4.77)$ \\
\hline
\end{tabular}

Table 6: Accuracy generated for the Dyntex++ database by different dynamic texture descriptors (represented in the rows) using the Random Forest, D-RVFL and LDA classifiers.

\begin{tabular}{l|c|c|c}
\hline Descriptor & Random Forest & D-RVFL & LDA \\
\hline VLBP & $84.47(0.26)$ & $79.48(0.48)$ & $89.54(0.35)$ \\
DPSWNet & $90.60(0.25)$ & $85.98(0.31)$ & $85.61(0.25)$ \\
DPSW & $90.17(0.08)$ & $63.13(0.42)$ & $83.22(0.17)$ \\
CNDT & $83.61(0.51)$ & $84.34(0.26)$ & $90.27(0.17)$ \\
Diffusion & $91.72(0.16)$ & $79.22(0.22)$ & $83.17(0.16)$ \\
RNN-DT & $95.37(0.10)$ & $48.22(0.34)$ & $88.89(0.28)$ \\
CPNN & $93.44(0.27)$ & $89.17(0.34)$ & $87.74(0.15)$ \\
\hline
\end{tabular}

as can be seen in Table 6. However, for the Random Forest and LDA classifiers, our results indicate that some classifiers may not be adapted to the proposed descriptor. Indeed, the performance of the classifiers depends on several issues such as the nature of the features, tuning of the parameters, etc. Thus, in some cases, simple classifiers can obtain best performances than more complex classifiers [70].

Table 7 shows the processing time needed, on average, to compute a feature vector from a single dynamic texture for each method. In the tests, we computed the average of 20 executions of feature extraction of a single sample using a 3.60 GHz Intel(R) Core i7, 64GB RAM, and 64-bit Operating System. The proposed method took $3.07 \mathrm{~s}$ and $2.47 \mathrm{~s}$ to extract the feature vector from the UCLA and Dyntex ++ database, respectively. The RNN-DT method took the lowest time, $1.35 \mathrm{~s}$ on the UCLA database and $1.83 \mathrm{~s}$ on the Dyntex++ database. Although the proposed method obtained the second-lowest time, the results demonstrate that our method is very competitive compared to the other approaches, taking a reasonable time to compute the features, and achieving high accuracies. 
Table 7: Computational processing time in seconds of the proposed method and other methods to compute the feature vector.

\begin{tabular}{lcc}
\hline Descriptor & UCLA & Dyntex++ \\
\hline VLBP & 3.67 & 2.60 \\
DPSW & 49.02 & 34.13 \\
DPSWNet & 8.97 & 48.78 \\
CNDT & 17.68 & 16.45 \\
Diffusion & 6.68 & 4.94 \\
RNN-DT & 1.35 & 1.83 \\
Proposed Method & 3.07 & 2.47 \\
\hline
\end{tabular}

\subsection{Dynamic Texture Segmentation}

Here, we show how our CPNN descriptors (described in Section 3) can be used for real-time dynamic texture segmentation. Experiments were done with videos captured from a moving boat on the Guerlédan lake in Brittany. In order to apply our descriptor for dynamic texture segmentation, we consider an approach based on overlapping blocks. Figure 7 summarizes the approach for dynamic texture segmentation: firstly, the video is divided into overlapping blocks; for each block a feature vector is obtained using our dynamic texture descriptor; finally the feature vector is labeled using our classification approach.

- Overlapping blocks: the dynamic texture video of $w \times h \times T$ pixels is divided into overlapping blocks $B$ of size $p \times p \times q$ pixels (as can be seen in Figure $7(\mathrm{a}))$. The blocks are evenly sampled using steps of size $l$ between each block (horizontally, vertically and temporally). The border pixels are not considered when it is not possible to fit a block.

- Feature extraction: a feature vector is obtained for each block of the dynamic texture video using the proposed method for dynamic texture description in Section 3

- Labeling: in this step, the blocks are labeled from their feature vector. For this, we use supervised classifiers. In this way, new blocks are predicted based on a classifier model trained using labeled blocks from other annotated videos. As we are using overlapping blocks, the pixels of the 


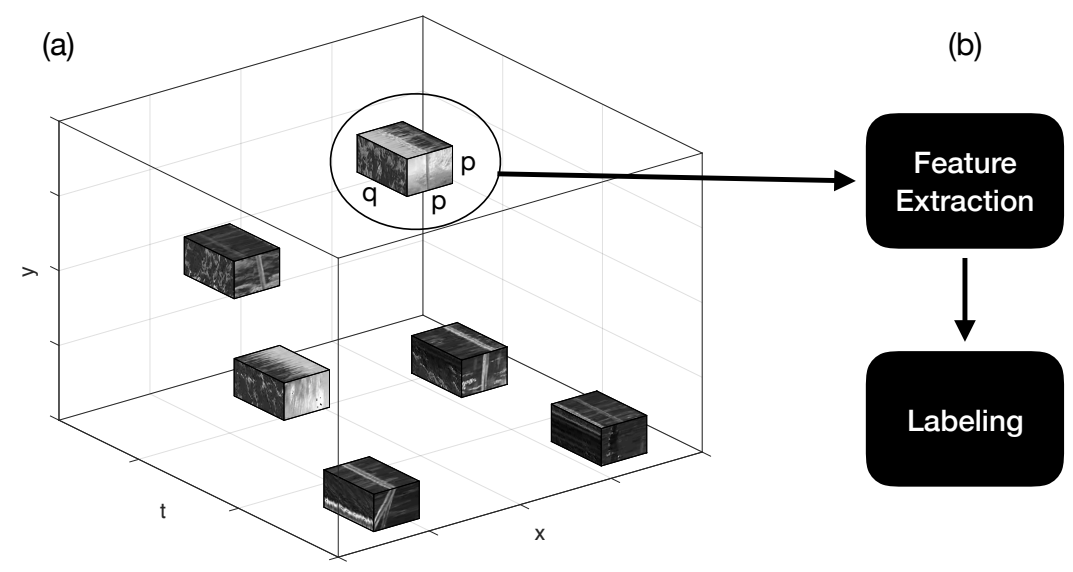

Figure 7: Illustration of the dynamic texture segmentation approach based on block labeling.

video belong to several blocks, thus their final label is provided by majority voting.

The goal is to test our dynamic texture descriptor to segment the different semantic regions of the video: water, sky and land (forest). Figure 8 (a) shows an illustration of the video used in the experiments. The main challenges of this type of video are the variability of textures due to perspective, the sun reflections, and of course, the motion of the boat. The video used in the training step has $288 \times 384 \times 1200$ pixels, while the video used in the testing step has $288 \times 384 \times 1621$ pixels. The labeled blocks used for the training of the classifier were obtained randomly from the different regions of the training video. In the experiments, we tested different sizes of step between blocks $(l=5,8,11)$ and a size of block equal to $p=q=30$ pixels. We also used two classifiers (Support Vector Machine (SVM) and 1-Nearest Neighbors (1-NN)) and different numbers of labeled samples to train the classifiers.

In Figure 9 is shown the first frame of the videos segmented into three classes, with each color representing a class: blue (sky), red (land) and transparent (water). In this figure, in the first column was used 1000 samples for training the 1-NN classifier and in the second column 2000 samples. Figure 10 shows the segmented videos using the SVM classifier. As can be seen, the proposed 


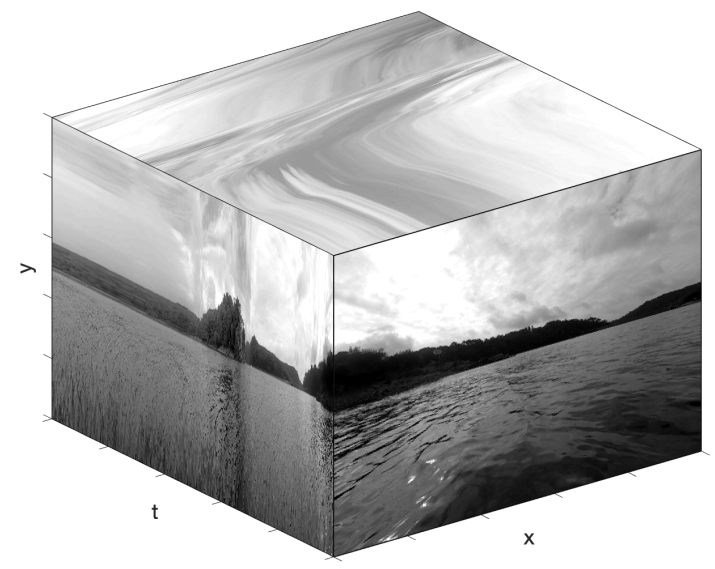

Figure 8: Space $\times$ time cuboid view of one moving boat video used in the experiment of segmentation.

scheme can identify well the three different classes in all examples. However, the method has more difficulties to delimit the border regions. To improve this point, we intend to investigate new ways of applying or refine the scheme for segmentation.

The main advantages of our method are the computational simplicity and the fast processing time, which makes the approach promising for real-time segmentation task and active learning. In addition, the proposed approach uses a small number of samples for training, which is another interesting characteristic for problems with few data samples.

\section{Conclusion}

In this paper, we present a method for dynamic texture recognition called CPNN, which learns a representation from the graph-based features using randomized neural networks. This is achieved through the adoption of a Complex Network framework for modeling a video through directed graphs, which are able to efficiently model the appearance and motion characteristics of the dynamic texture. From this, graph-based features can be learned by the randomized 


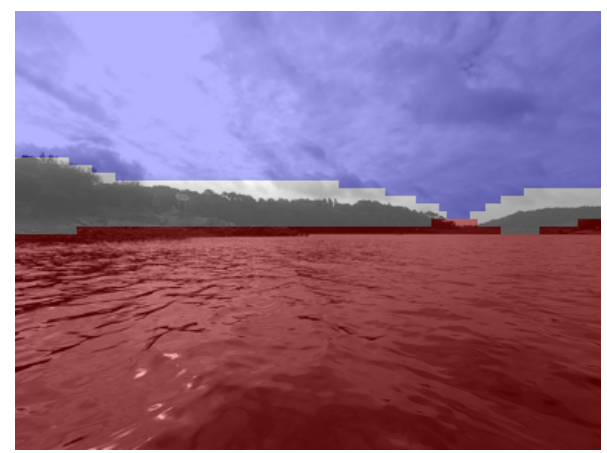

(a) $l=5$

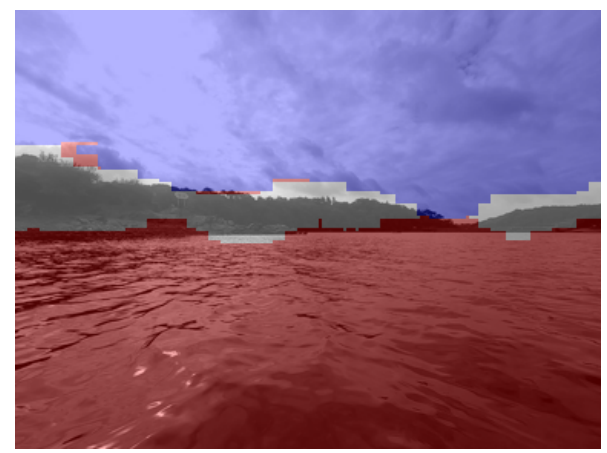

(c) $l=8$

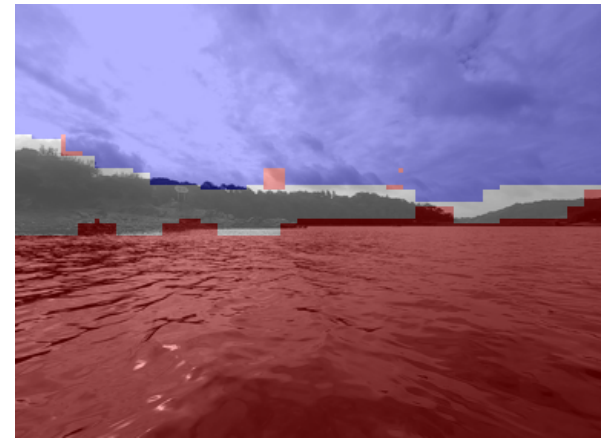

(e) $l=11$

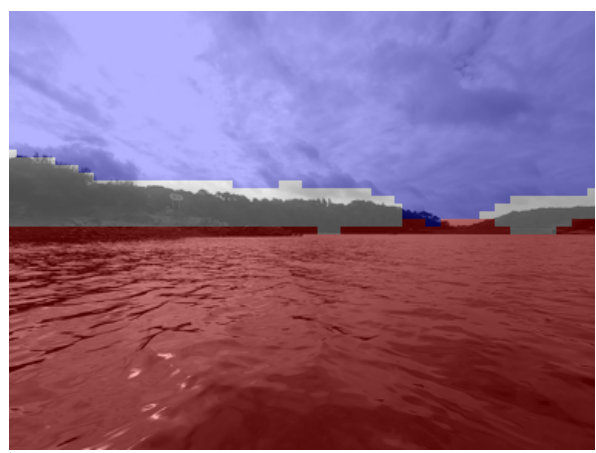

(b) $l=5$

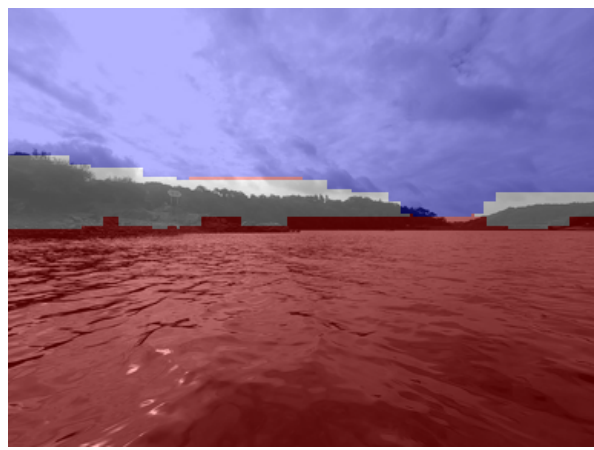

(d) $l=8$

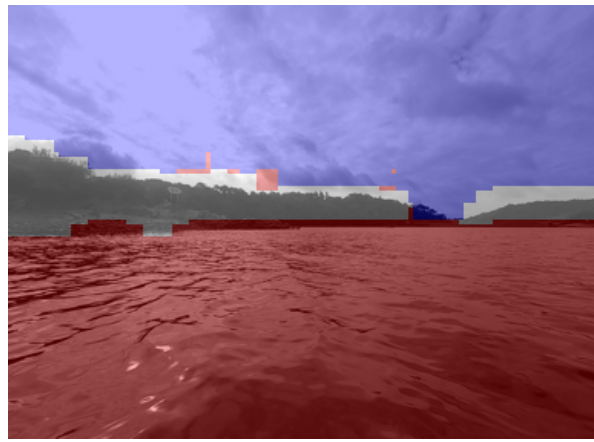

(f) $l=11$

Figure 9: One frame of segmented video using our DT model with the KNN classifier, for different values of step parameter $l$, and different numbers of training samples (left: 1000, right: 2000). 


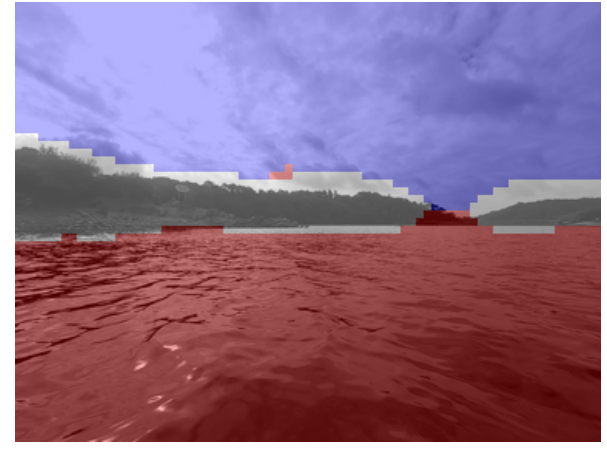

(a) $l=5$

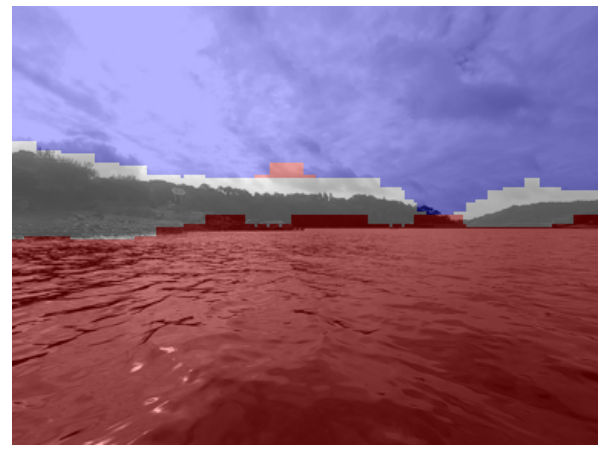

(c) $l=8$

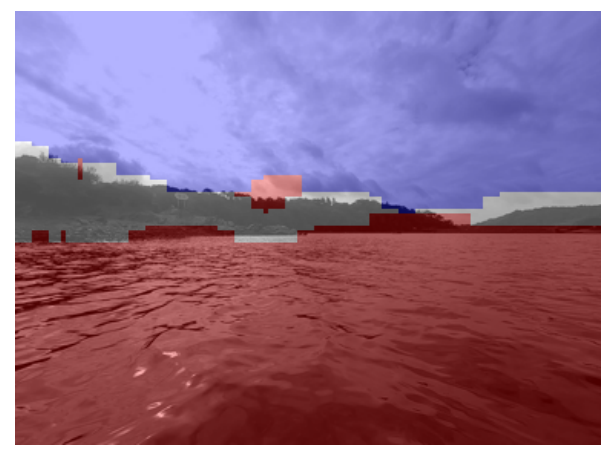

(e) $l=11$

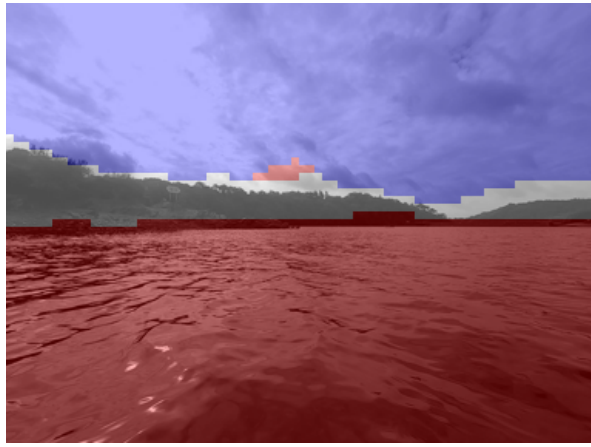

(b) $l=5$

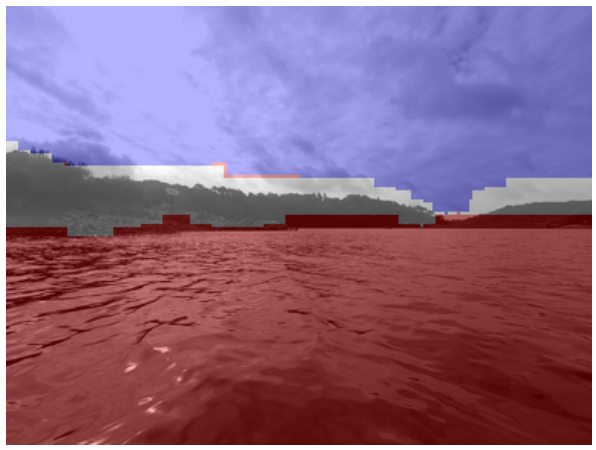

(d) $l=8$

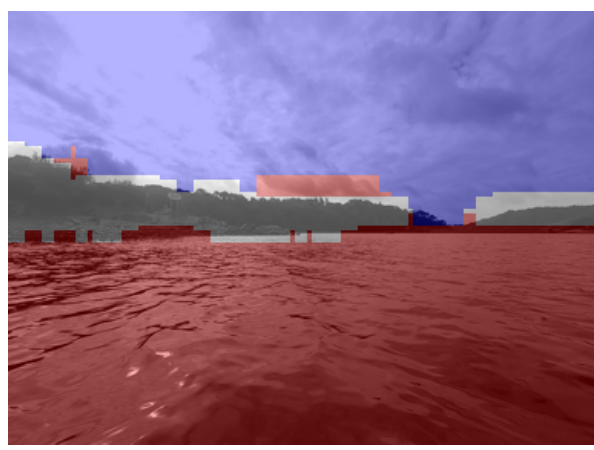

(f) $l=11$

Figure 10: Same as Figure 9 but using the SVM classifier. 
neural network, which has a simple and fast learning algorithm, producing a representative feature vector through the trained weights of the output layer. Based on the experiments, we adopted in the proposed method six randomized neural networks of 1 hidden layer with 4, 24, and 29 number of hidden units and input layers of size 4 and 10 features.

We have tested the CPNN method on two benchmarks for the task of dynamic texture classification. The results lead to the conclusion that our method provides discriminative dynamic texture descriptors using a simple classifier. Also, experiments of computational processing time demonstrated a competitive performance of the proposed method compared to the others. Based on these findings and on some experiments in dynamic texture segmentation, the proposed method can be a valuable tool for real-time applications and could be investigated for active learning purposes. These observations motivate the future investigation of exploring the complex network frameworks and learning methods for dynamic texture analysis. As limitation and future work, new manners of creating the training set of the neural network can be explored, such as the use of clustering measures, hierarchical degree and joint-degree of the vertices. The interpretation of the physical meaning of the learned features by the proposed model should also be further investigated. Another research issue is to investigate other neural network architectures with more layers (e.g., D-RVFL) to learn the features, although computational time may increase. Furthermore, our method learns the features using a regression model from RNN. However, we believe that a classification model in which each output neuron represents one class can be employed to learn the features and improve the results.

\section{Acknowledgments}

Lucas C. Ribas gratefully acknowledges the financial support grant \#2016/237638 and \#2019/03277-0, São Paulo Research Foundation (FAPESP). Jarbas Joaci de Mesquita Sá Junior thanks CNPq (National Council for Scientific and Technological Development, Brazil) (Grant: 302183/2017-5) for the financial sup- 
port of this work. O. M. Bruno acknowledges support from CNPq (Grant \#307897/2018-4) and FAPESP (grant \#2014/08026-1, 2018/22214-6 and 2016/18809-

9). The authors wish to thank Thomas Simon and Clément Yver for allowing them to use their videos from Guerlédan's lake.

\section{References}

[1] G. Doretto, A. Chiuso, Y. Wu, S. Soatto, Dynamic textures, International Journal of Computer Vision 51 (2) (2003) 91-109.

[2] Y. Xu, Y. Quan, H. Ling, H. Ji, Dynamic texture classification using dynamic fractal analysis, in: 2011 International Conference on Computer Vision, 2011, pp. 1219-1226.

[3] L. C. Ribas, W. N. Gonçalves, O. M. Bruno, Dynamic texture analysis with diffusion in networks, Digital Signal Processing 92 (2019) 109-126.

[4] X. Zhao, Y. Lin, J. Heikkil, Dynamic texture recognition using volume local binary count patterns with an application to 2D face spoofing detection, IEEE Transactions on Multimedia 20 (3) (2018) 552-566.

[5] K. G. Derpanis, R. P. Wildes, Classification of traffic video based on a spatiotemporal orientation analysis, in: IEEE Workshop on Applications of Computer Vision (WACV), 2011, pp. 606-613.

[6] W. Li, V. Mahadevan, N. Vasconcelos, Anomaly detection and localization in crowded scenes, IEEE Transactions on Pattern Analysis and Machine Intelligence 36 (1) (2014) 18-32.

[7] K. Dimitropoulos, P. Barmpoutis, N. Grammalidis, Spatio-temporal flame modeling and dynamic texture analysis for automatic video-based fire detection, IEEE Transactions on Circuits and Systems for Video Technology 25 (2) (2015) 339-351. 
[8] R. P. Wildes, J. R. Bergen, Qualitative spatiotemporal analysis using an oriented energy representation, in: European Conference on Computer Vision, Springer, 2000, pp. 768-784.

[9] K. G. Derpanis, R. P. Wildes, Dynamic texture recognition based on distributions of spacetime oriented structure, in: 2010 IEEE Computer Society Conference on Computer Vision and Pattern Recognition, IEEE, 2010, pp. 191-198.

[10] S. R. Arashloo, J. Kittler, Dynamic Texture Recognition Using Multiscale Binarized Statistical Image Features, IEEE Transactions on Multimedia 16 (8) (2014) 2099-2109.

[11] C. Feichtenhofer, A. Pinz, R. P. Wildes, Bags of spacetime energies for dynamic scene recognition, in: Proceedings of the IEEE Conference on Computer Vision and Pattern Recognition, 2014, pp. 2681-2688.

[12] A. R. Rivera, O. Chae, Spatiotemporal directional number transitional graph for dynamic texture recognition, IEEE Transactions on Pattern Analysis and Machine Intelligence 37 (10) (2015) 2146-2152.

[13] T. T. Nguyen, T. P. Nguyen, F. Bouchara, Prominent local representation for dynamic textures based on high-order Gaussian-gradients, IEEE Transactions on Multimedia.

[14] X. Zhao, Y. Lin, L. Liu, J. Heikkilä, W. Zheng, Dynamic texture classification using unsupervised 3d filter learning and local binary encoding, IEEE Transactions on Multimedia 21 (7) (2019) 1694-1708.

[15] T. T. Nguyen, T. P. Nguyen, F. Bouchara, A novel filtering kernel based on difference of derivative Gaussians with applications to dynamic texture representation, Signal Processing: Image Communication 98 (2021) 116394.

[16] R. Polana, R. Nelson, Temporal texture and activity recognition, in: M. Shah, R. Jain (Eds.), Motion-Based Recognition, Vol. 9 of Computational Imaging and Vision, Springer Netherlands, 1997, pp. 87-124. 
[17] C.-H. Peh, L.-F. Cheong, Synergizing spatial and temporal texture, IEEE Transactions on Image Processing 11 (10) (2002) 1179-1191.

[18] R. Péteri, D. Chetverikov, Dynamic texture recognition using normal flow and texture regularity, in: Pattern Recognition and Image Analysis, Springer, 2005, pp. 223-230.

[19] S. Fazekas, D. Chetverikov, Dynamic texture recognition using optical flow features and temporal periodicity, in: International Workshop on ContentBased Multimedia Indexing (CBMI), 2007, pp. 25-32.

[20] T. T. Nguyen, T. P. Nguyen, F. Bouchara, X. S. Nguyen, Directional beams of dense trajectories for dynamic texture recognition, in: International Conference on Advanced Concepts for Intelligent Vision Systems, Springer, 2018, pp. $74-86$.

[21] L. N. Couto, C. A. Barcelos, Singular patterns in optical flows as dynamic texture descriptors, in: Iberoamerican Congress on Pattern Recognition, Springer, 2018, pp. 351-358.

[22] G. Zhao, M. Pietikainen, Dynamic texture recognition using local binary patterns with an application to facial expressions, IEEE Transactions on Pattern Analysis and Machine Intelligence 29 (6) (2007) 915-928.

[23] G. Zhao, M. Pietikäinen, Dynamic texture recognition using volume local binary patterns, in: Dynamical Vision, Springer Berlin Heidelberg, 2006, pp. $165-177$.

[24] R. D. Tiwari, V. Tyagi, Dynamic texture recognition using multiresolution edge-weighted local structure pattern, Computers \& Electrical Engineering 62 (2017) 485-498.

[25] D. Tiwari, V. Tyagi, A novel scheme based on local binary pattern for dynamic texture recognition, Computer Vision and Image Understanding 150 (2016) 58-65. 
[26] G. Zhao, T. Ahonen, J. Matas, M. Pietikainen, Rotation-invariant image and video description with local binary pattern features, IEEE transactions on image processing 21 (4) (2011) 1465-1477.

[27] J. Luo, Z. Tang, H. Zhang, Y. Fan, Y. Xie, Ltgh: A dynamic texture feature for working condition recognition in the froth flotation, IEEE Transactions on Instrumentation and Measurement 70 (2021) 1-10.

[28] T. T. Nguyen, T. P. Nguyen, F. Bouchara, X. S. Nguyen, Momental directional patterns for dynamic texture recognition, Computer Vision and Image Understanding 194 (2020) 102882.

[29] B. Raman, D. Sadhya, et al., Dynamic texture recognition using local tetra pattern-three orthogonal planes (LTrP-TOP), The Visual Computer 36 (3) (2020) 579-592.

[30] D. Tiwari, V. Tyagi, Dynamic texture recognition based on completed volume local binary pattern, Multidimensional Systems and Signal Processing 27 (2) (2016) 563-575.

[31] X. Qi, C.-G. Li, G. Zhao, X. Hong, M. Pietikäinen, Dynamic texture and scene classification by transferring deep image features, Neurocomputing 171 (2016) 1230-1241.

[32] S. R. Arashloo, M. C. Amirani, A. Noroozi, Dynamic texture representation using a deep multi-scale convolutional network, Journal of Visual Communication and Image Representation 43 (2017) 89-97.

[33] V. Andrearczyk, P. F. Whelan, Convolutional neural network on three orthogonal planes for dynamic texture classification, Pattern Recognition 76 (2018) 36-49.

[34] X. Zhao, Y. Lin, L. Liu, Dynamic texture recognition using 3d random features, in: ICASSP 2019-2019 IEEE International Conference on Acoustics, Speech and Signal Processing (ICASSP), IEEE, 2019, pp. 2102-2106. 
[35] J. J. M. Sá Junior, L. C. Ribas, O. M. Bruno, Randomized neural network based signature for dynamic texture classification, Expert Systems with Applications 135 (2019) 194-200.

[36] A. Ravichandran, R. Chaudhry, R. Vidal, View-invariant dynamic texture recognition using a bag of dynamical systems, in: 2009 IEEE Conference on Computer Vision and Pattern Recognition, 2009, pp. 1651-1657.

[37] A. Ravichandran, R. Chaudhry, R. Vidal, Categorizing dynamic textures using a bag of dynamical systems, IEEE Transactions on Pattern Analysis and Machine Intelligence 35 (2) (2012) 342-353.

[38] Y. Wang, S. Hu, Chaotic features for dynamic textures recognition, Soft Computing 20 (5) (2016) 1977-1989.

[39] W. N. Gonçalves, O. M. Bruno, Dynamic texture analysis and segmentation using deterministic partially self-avoiding walks, Expert Systems with Applications 40 (11) (2013) 4283 - 4300.

[40] W. N. Gonçalves, B. B. Machado, O. M. Bruno, A complex network approach for dynamic texture recognition, Neurocomputing 153 (2015) 211 220.

[41] L. C. Ribas, J. J. M. Sá Junior, L. F. S. Scabini, O. M. Bruno, Fusion of complex networks and randomized neural networks for texture analysis, Pattern Recognition 103 (2020) 107189.

[42] L. C. Ribas, O. M. Bruno, Dynamic texture analysis using networks generated by deterministic partially self-avoiding walks, Physica A: Statistical Mechanics and its Applications 541 (2020) 122105.

[43] A.-L. Barabási, R. Albert, Emergence of scaling in random networks, science 286 (5439) (1999) 509-512.

[44] M. Girvan, M. E. Newman, Community structure in social and biological networks, Proceedings of the national academy of sciences 99 (12) (2002) 7821-7826. 
[45] D. J. Watts, S. H. Strogatz, Collective dynamics of small-world networks, nature 393 (6684) (1998) 440-442.

[46] L. F. Scabini, R. H. Condori, W. N. Gonçalves, O. M. Bruno, Multilayer complex network descriptors for color-texture characterization, Information Sciences 491 (2019) 30-47.

[47] W. F. Schmidt, M. A. Kraaijveld, R. P. W. Duin, Feedforward neural networks with random weights, in: Proceedings., 11th IAPR International Conference on Pattern Recognition. Vol.II. Conference B: Pattern Recognition Methodology and Systems, 1992, pp. 1-4.

[48] Y.-H. Pao, Y. Takefuji, Functional-link net computing: theory, system architecture, and functionalities, Computer 25 (5) (1992) 76-79.

[49] Y.-H. Pao, G.-H. Park, D. J. Sobajic, Learning and generalization characteristics of the random vector functional-link net, Neurocomputing 6 (2) (1994) 163-180.

[50] G.-B. Huang, Q.-Y. Zhu, C.-K. Siew, Extreme learning machine: theory and applications, Neurocomputing 70 (1) (2006) 489-501.

[51] E. H. Moore, On the reciprocal of the general algebraic matrix, Bulletin of the American Mathematical Society 26 (1920) 394-395.

[52] R. Penrose, A generalized inverse for matrices, Mathematical Proceedings of the Cambridge Philosophical Society 51 (3) (1955) 406-413.

[53] A. N. Tikhonov, On the solution of ill-posed problems and the method of regularization, Dokl. Akad. Nauk USSR 151 (3) (1963) 501-504.

[54] D. Calvetti, S. Morigi, L. Reichel, F. Sgallari, Tikhonov regularization and the L-curve for large discrete ill-posed problems, Journal of Computational and Applied Mathematics 123 (1) (2000) 423 - 446.

[55] D. H. Lehmer, Mathematical methods in large scale computing units, Annals Comp. Laboratory Harvard University 26 (1951) 141-146. 
[56] S. K. Park, K. W. Miller, Random number generators: good ones are hard to find, Communications of the ACM 31 (10) (1988) 1192-1201.

[57] J. J. M. Sá Junior, A. R. Backes, ELM based signature for texture classification, Pattern Recognition 51 (2016) 395-401.

[58] B. Ghanem, N. Ahuja, Maximum margin distance learning for dynamic texture recognition, in: Proceedings of the 11th European Conference on Computer Vision: Part II, ECCV'10, Springer-Verlag, Berlin, Heidelberg, 2010, pp. 223-236.

[59] R. Péteri, S. Fazekas, M. J. Huiskes, DynTex: A comprehensive database of dynamic textures, Pattern Recognition Letters 31 (12) (2010) 1627-1632.

[60] P. Saisan, G. Doretto, Y. N. Wu, S. Soatto, Dynamic texture recognition, in: Proceedings of the 2001 IEEE Computer Society Conference on Computer Vision and Pattern Recognition. CVPR 2001, Vol. 2, 2001, pp. II-58-II-63 vol.2.

[61] G. Holmes, A. Donkin, I. H. Witten, Weka: A machine learning workbench, in: Proceedings of ANZIIS'94-Australian New Zealand Intelligent Information Systems Conference, IEEE, 1994, pp. 357-361.

[62] F. Wilcoxon, Individual comparisons by ranking methods, in: Breakthroughs in statistics, Springer, 1992, pp. 196-202.

[63] A. B. Chan, N. Vasconcelos, Classifying video with kernel dynamic textures, in: IEEE Conference on Computer Vision and Pattern Recognition (CVPR), 2007, pp. 1-6.

[64] Y. Xu, S. Huang, H. Ji, C. Fermüller, Scale-space texture description on SIFT-like textons, Computer Vision and Image Understanding 116 (9) (2012) 999-1013.

[65] Y. Wang, S. Hu, Exploiting high level feature for dynamic textures recognition, Neurocomputing 154 (2015) 217-224. 
[66] H. Ji, X. Yang, H. Ling, Y. Xu, Wavelet domain multifractal analysis for static and dynamic texture classification, IEEE Transactions on Image Processing 22 (1) (2013) 286-299.

[67] L. Breiman, Random forests, Machine learning 45 (1) (2001) 5-32.

[68] R. Katuwal, P. N. Suganthan, Stacked autoencoder based deep random vector functional link neural network for classification, Applied Soft Computing 85 (2019) 105854.

[69] R. A. Fisher, The use of multiple measurements in taxonomic problems, Annals of Eugenics 7 (7) (1936) 179-188.

[70] D. R. Amancio, C. H. Comin, D. Casanova, G. Travieso, O. M. Bruno, F. A. Rodrigues, L. da Fontoura Costa, A systematic comparison of supervised classifiers, PloS one 9 (4) (2014) e94137. 